\title{
ABSORPTION SPECTRA OF IRON, COBALT, AND NICKEL
}

\author{
By W. F. Meggers and F. M. Walters, jr.
}

The underwater spark absorption spectra, as well as the ordinary vapor absorptions, have been investigated by others, but not in sufficient detail in connection with the spectral structures of $\mathrm{Fe}, \mathrm{Co}$, and $\mathrm{Ni}$. Employing high potential condensed sparks between electrodes of iron, cobalt, and nickel immersed in water, the underwater spark spectra of these metals were reexamined throughout the visible and ultra-violet regions. The spectrograms show 265 iron lines (2166 to $4404 \mathrm{~A}$ ), 340 cobalt lines ( 2137 to $4121 \mathrm{~A}$ ), and 225 nickel lines (2124 to $3858 \mathrm{~A}$ ) absorbed in the source. In each case the majority of these are identical with the stronger lines of the arc-emission spectra, and practically all such lines are found to involve either the normal state or some low metastable state of the neutral atoms. These results confirm and extend the known spectral structures for neutral $\mathrm{Fe}, \mathrm{Co}$, and $\mathrm{Ni}$; the normal states of these atoms are represented by ${ }^{5} \mathrm{D},{ }^{4} \mathrm{~F}$, and ${ }^{3} \mathrm{~F}$ terms, respectively. The type of source used showed most of the metallic spark lines in emission, but certain groups were present in absorption with low intensity. The latter involve low energy states of the ionized atoms; these normal or metastable states are represented by ${ }^{6} \mathrm{D},{ }^{5} \mathrm{~F}$, and ${ }^{4} \mathrm{~F}$ terms for ionized $\mathrm{Fe}, \mathrm{Co}$, and $\mathrm{Ni}$, respectively.

\section{CONTENTS}

I. Introduction

II. Underwater spark spectra_.

III. Apparatus and experimental details _.

IV. Results_...-.

1. Iron $(\mathrm{Fe}=55.84 ; \mathrm{Z}=26)$

2. Cobalt $(\mathrm{Co}=58.97 ; \mathrm{Z}=27) \ldots$

3. Nickel $(\mathrm{Ni}=58.68 ; \mathrm{Z}=28) \ldots \ldots$

V. Discussion_..._.

VI. Bibliography _........ 226

\section{INTRODUCTION}

Atoms in the vapor state emit radiation if they return from excited to lower energy values, and they may absorb energy from a continuous spectrum in the reverse process. The absorption lines occupy the same positions otherwise occupied by characteristic bright lines of the emission spectrum. Moreover, if the absorbing atoms are unexcited at the beginning they are capable of absorbing those lines which involve the energy change from the zero or normal state to some immediately excited state. In this fact lies the importance of absorption data for the analysis of spectral structures; they determine the order of the spectral terms and identify the one which describes the normal state of the atom. 
The ideal experimental arrangement is one in which a continuous spectrum is photographed after the light has passed through a tube of metal vapor at a temperature to produce sufficient vapor pressure without appreciable excitation of the atoms. The continuous spectrum is then crossed by absorption lines which are coincident with prominent lines in the emission spectrum of the electric arc. Such experiments with the alkali metals $(1)^{1}$ have long been familiar and they have recently been performed with other elements (2) in connection with the analysis of more complex spectra. In particular the absorption spectra of iron, cobalt, and nickel were investigated in this manner by Angerer and Joos (3).

Metals such as those under discussion and others with very high boiling points are vaporized and studied only with great difficulty, so that easier methods of obtaining the same results have been sought. Under certain conditions the continuous spectrum produced when condensed high-voltage discharges pass between metallic electrodes submerged under water is interrupted by absorption lines characteristic of the metal, and these phenomena have laterly received considerable attention in connection with the analysis of complex spectra. The purpose of this paper is to report on our observations of the underwater spark absorption spectra of iron, cobalt, and nickel to show how these data confirm the known structures of the emission spectra and to present some extensions of the structural analyses.

\section{UNDERWATER SPARK SPECTRA}

The spectral characteristics of sparks between metallic electrodes immersed in liquids have been under investigation for a quarter of a century. The first experiments, those of Wilsing (4), of Lockyer (5), and of Hale (6), were inspired by problems in stellar spectra and were concerned mainly with spectral line displacements due to pressure and other causes. Extensive investigations with various metals were carried out by Konen (7), Finger (8), E. and L. Bloch (9), and Hulburt (10). Konen gave wave length data for $\mathrm{Fe}, \mathrm{Al}$, and $\mathrm{Cu}$, and was the first to identify certain $\mathrm{H}_{2} \mathrm{O}$ absorption bands which always occur in the spectra of underwater sparks. Finger observed that lines belonging to a spectral series behaved in the same way in respect to changes occuring in underliquid sparks. This rule was supported by his results with $\mathrm{Cu}, \mathrm{Ag}, \mathrm{Ca}, \mathrm{Zn}, \mathrm{Cd}, \mathrm{Al}$, and $\mathrm{Tl}$. Similar conclusions were drawn from the detailed observations of $\mathrm{E}$. and $\mathrm{L}$. Bloch, who photographed the underwater spark spectra (5000 to $2200 \mathrm{~A}$ ) of $\mathrm{Zn}, \mathrm{Cd}, \mathrm{Hg}, \mathrm{Mg}, \mathrm{Ca}, \mathrm{Cu}, \mathrm{Ag}, \mathrm{Al}, \mathrm{Tl}, \mathrm{Sn}, \mathrm{Pb}, \mathrm{Bi}, \mathrm{Sb}$, and Fe. They found that, in general, two groups of lines, reversed and bright, appeared, the former being representative of the arc spectrum and the latter of the spark spectrum. In addition to series lines in

1 The figures given in parentheses here and throughout the text relate to the reference numbers in the bibliography at the end of this paper. 
arc spectra, the so-called "single line" (intersystem combination or resonance line) was always absorbed. The suggestion was made that such observations might facilitate the search for spectral regularities.

In Hulburt's experiments the absorption lines were identified in the spectral region 4500 to $2000 \mathrm{~A}$, those appearing in the underwater spark spectra of $\mathrm{Al}, \mathrm{Bi}, \mathrm{Cd}, \mathrm{Au}, \mathrm{Ir}, \mathrm{Pb}, \mathrm{Mg}, \mathrm{Pt}, \mathrm{Rh}, \mathrm{Ag}, \mathrm{Sn}, \mathrm{Zn}$ were those which were reversed in the arc, no more and no less. For the metals $\mathrm{Sb}, \mathrm{Co}, \mathrm{Cr}, \mathrm{Cu}, \mathrm{Fe}, \mathrm{Mo}, \mathrm{Ni}$, and $\mathrm{W}$ all the lines reversed in the arc appeared as absorption lines, and in addition, the underwater spark spectra of these elements exhibited altogether more than 400 absorption lines which are not listed as reversed in the arc, but complete details are not given for these.

An investigation of the physical and electrical conditions determining the characteristics of underwater sparks was recently made by Miss Stücklen (11), who studied the effect on Cd spectra of varying $(a)$ external spark gap, 0.5 to $3 \mathrm{~cm}$, (b) self-induction to change wave length from 300 to $1,100 \mathrm{~m}$, and $(c)$ diameter of electrodes, 3 to $8 \mathrm{~mm}$, and concluded that the appearance of absorption lines was favored by increasing the frequency, decreasing the potential, and increasing the diameter of the electrodes. Under the conditions of Miss Stücklen's experiment the fundamental spark lines of $\mathrm{Cd}$ appeared also in absorption.

During the past year several attempts have been made (12) to correlate the regularities of complex spectra with absorption observations in the underwater spark; some of these will be referred to again in connection with the results of the present investigation.

A general survey of all the published data on lines absorbed in underwater spark spectra of various elements gives the impression that results obtainable by this method are more comparable with those of true absorption experiments than might be expected, since the simultaneous appearance of spark lines in emission is evidence that at least some of the atoms in such a source must be ionized. In addition to fundamental arc lines which are completely and symmetrically absorbed in the underwater spark, some of the lines involving the normal state of ionized atoms are indeed usually detected in the same source, although they are of relatively low intensity. It appears that a large majority of the atoms in the outer envelope of the spark are in the normal state, a smaller number are in low metastable states, and a few are ionized; but it may be concluded that the conditions in this source are not favorable to the production of the remaining intermediate energy states, since they play no part in the absorption phenomena.

The lines observed as absorbed in the underwater spark are also remarkably in accord with other observations which have been demonstrated to be very significant in identifying the low levels of energy. 
1. All the lines observed as "reversals" in arc emission spectra are always observed as absorption lines in underwater spark spectra and sometimes many more.

2. Insofar as temperature classifications of emission lines have been studied in the electric furnace, it is evident that the lines ordinarily absorbed in the underwater spark spectra are always the lines of lowest temperature classes, those first to appear as the furnace temperature rises.

In the determination of absorption spectra by means of the underwater spark, two respects in which this method imposes limitations on the completeness of the results must be mentioned.

1. Bands assigned to water vapor always appear in absorption, and these may occasionally obscure absorbed lines characteristic of the metal electrodes. The band at $3063 \mathrm{~A}$ is especially prominent; it extends nearly to $3200 \mathrm{~A}$ and contains a large number of lines. This difficulty can be overcome by using some other liquid.

2. It has frequently been mentioned that the lines (both emission and absorption) in underwater spark spectra are widened, some are diffuse, and certain ones are displaced as compared with their appearance in ordinary sources. These effects make it difficult to resolve lines which are very close and sometimes lead to an uncertainty in identifying lines which appear absorbed.

\section{APPARATUS AND EXPERIMENTAL DETAILS}

The great violence of the powerful electric discharge between electrodes under water showed, in preliminary experiments with improvised apparatus, that it was necessary to build a rugged device for holding and adjusting the metal electrodes. The apparatus constructed for this purpose is shown in Figure 1. It consists of a block of bakelite to which the two electrode holders are attached. To adjust the electrodes so that they are opposite each other and the proper distance apart, the lower holder is adjustable horizontally and either or both of them may be moved up or down by means of one-half $\mathrm{mm}$ pitch screws, the latter motion being permitted, and frequently required, during the operation of the spark. This apparatus was placed in a wooden box of about 3 liters capacity, opposite sides of which were furnished with quartz windows. The spark was operated about 5 or $6 \mathrm{~cm}$ under water and about $2 \mathrm{~cm}$ from the window. Tap water flowed through the box continuously at the rate of 1 to 2 liters per minute to carry off the colloidal metal, which would otherwise pollute the water and render it less transparent.

A 40,000-volt transformer was supplied with 10 to 12 amperes, 60 cycle, alternating current at 110 volts, and the secondary was connected to the underwater spark with an adjustable air gap in series and some condensers of $0.006 \mu \mathrm{f}$ capacity in parallel. The 
Scientific Papers of the Bureau of Standards, Vol. 22

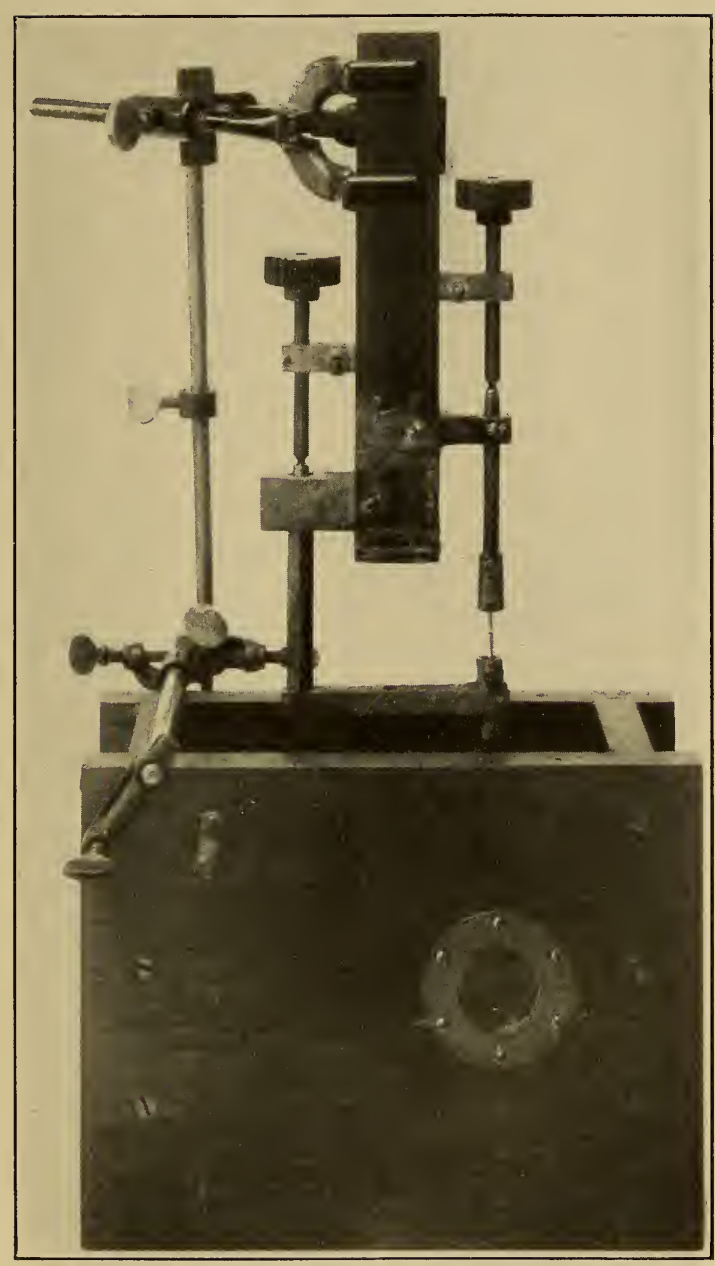

FIg. 1.-Underwater-spark apparatus 


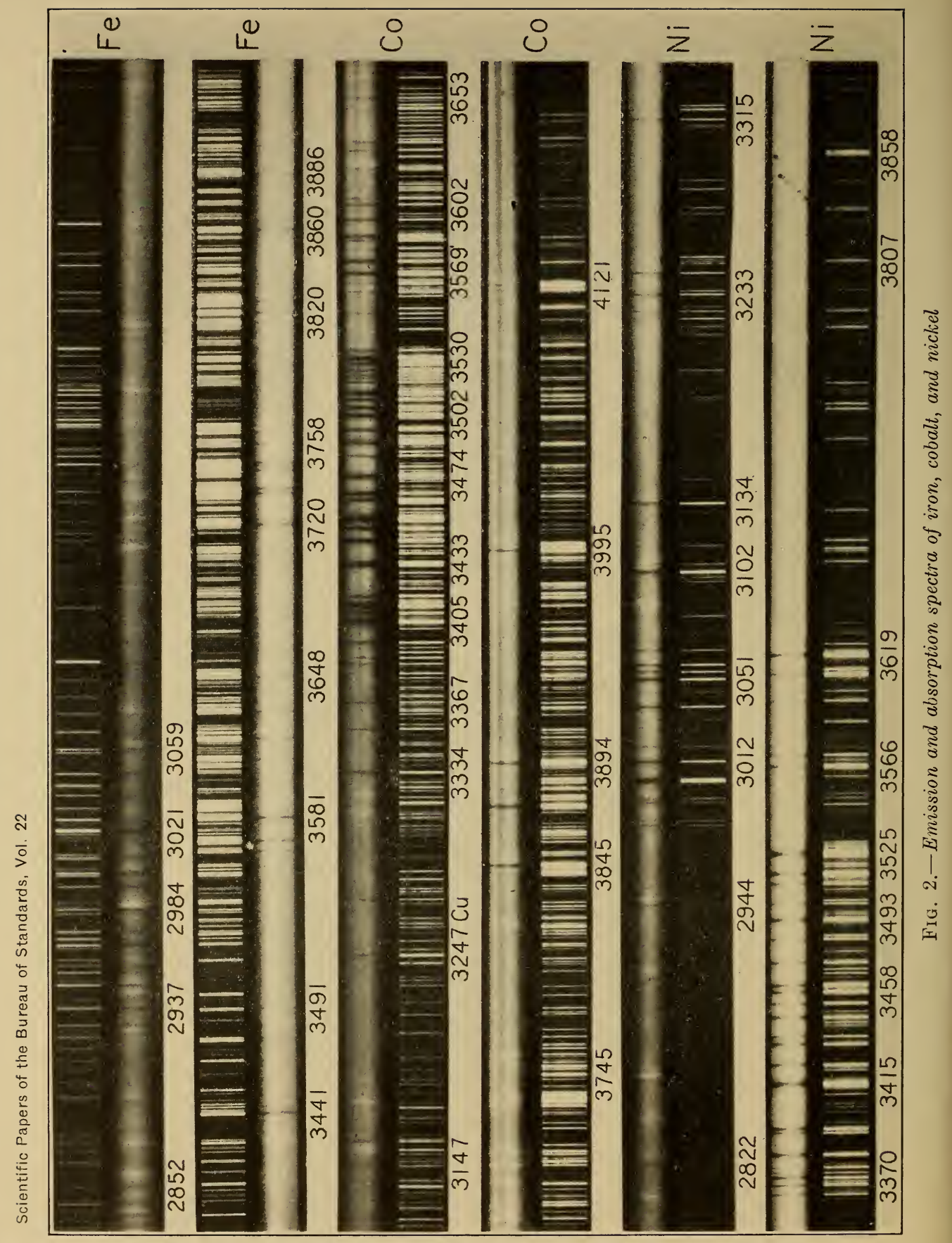


external spark gap was between zinc cylinders about $10 \mathrm{~mm}$ apart, and the distance between the electrodes of the underwater spark was about one-half $\mathrm{mm}$. Under the conditions of our arrangement no marked effect assignable to the diameter of the electrodes was observed. For the most part ingots averaging $10 \mathrm{~mm}^{2}$ cross section were employed.

An image of the underwater spark was focussed with a quartz condenser upon the slit of the spectrograph. Our concave gratings were used for observations in the visible and ultra-violet to $2400 \mathrm{~A}$, below which a Hilger quartz spectrograph with lenses of about $60 \mathrm{~cm}$ focal length was employed. The exposures were usually only a few minutes, but about an hour was required to extend the spectra below $2300 \mathrm{~A}$. Ordinary photographic plates were used except for the shortest waves, which were recorded on Schumann plates supplied by Hilger.

As has been shown by others, there are usually several types of lines to be distinguished; $(a)$ emission lines, usually broadened and displaced, (b) symmetrical absorption lines, and (c) partial absorption, very unsymmetrical. The first class belongs to the spectrum of the ionized atom and is of no particular interest here. The latter classes, being characteristic of arc spectra, were carefully identified and are recorded in the following tables. The partial reversals are always on the violet side of the emission lines; they are designated $p$ because of a suspicion that they might be penultimate lines in the sense that this word was proposed by Russell. It appears that such lines actually involve some one of the low metastable states, while lines actually associated with the normal state are broad and symmetrical reversals. These three classes of lines are illustrated in Figure 2, where portions of the underwater spark spectra and of the arc emission spectra of $\mathrm{Fe}, \mathrm{Co}$, and $\mathrm{Ni}$ are reproduced.

\section{RESULTS}

For each of the three elements-iron, cobalt, and nickel-we give first a table of absorbed lines and their classifications and then a table of energy levels. In the first type of table the data presented in succeeding columns are wave lengths $(\lambda)$, estimated intensities in absorption (A) and emission (E), vacuum wave numbers ( $\nu$, term combinations, and notes. Wave numbers were calculated from Kayser's Tabelle der Schwingungszahlen. The arc emission intensities accompanied by Roman numerals indicating the temperature classes are quoted from King. In the last column the lines observed as absorbed by Angerer and Joos (A) and by Gieseler and Grotrian $(\mathrm{G})$ are noted. The notation for term combinations is the one which is now in common use for the description of spectral regularities; the letters $\mathrm{S}, \mathrm{P}, \mathrm{D}, \mathrm{F}, \mathrm{G}$ correspond to quantum num- 
bers $l=0,1,2,3,4$, respectively; the superscript indicates the maximum multiplicity $r$ of the system and the subscript is the inner quantum number $j$ associated with the energy level. The tables of terms present values of all the known low energy levels calculated on the basis of zero energy for the lowest level which represents the normal state of the atom. The relative values of the higher levels referred to in the first type of table can be derived from the combinations, since the wave number of any line is represented by the difference of two levels, one of which in each case belongs to the low set listed in the second type of table. Electron configurations which on Hund's (13) theory are responsible for the various low energy terms are indicated in the last column of the term tables.

\section{IRON $(\mathrm{Fe}=55.84 ; Z=26)$}

Cylindrical rods of iron, $3 \mathrm{~mm}$ diameter, were used as electrodes for the underwater spark. No impurities were detected spectrographically. The wave lengths shorter than $2373 \mathrm{~A}$ are quoted from Schumacher (14); the longer ones from Burns (15). To the violet of $3900 \mathrm{~A}$ a considerable number of the stronger iron lines are readily observed as partial reversals in the ordinary arc emission spectrum, and together with King's (16) temperature data these observations were of importnce in the detection of the first regularities among the arc lines of iron (17). The structural analysis of this spectrum was presented in more detail in subsequent papers (18). The term combinations in Table 1 are essentially those given in Laporte's papers, except that the notation has been modified to conform with recent practice and some new levels have been added.

Angerer and Joos (3) observed about 100 iron lines in absorption, 49 of which were recognized as involving levels of a quintet-D term assumed to represent the normal state of the iron atom. That this term describes the normal state of the Fe atom was also concluded by Gieseler and Grotrian (19), who observed 11 lines in absorption.

The underwater spark absorption spectrum of iron has recently been studied by Sur (12), who discussed his observations in connection with Laporte's analysis. He added nothing to this analysis, but believed that it was not complete with respect to the low energy levels. His objections were answered by Laporte (20), and it is now generally conceded that no terms of lower energy than $a^{5} \mathrm{D}$ are to be expected in this spectrum. Sur recorded 215 lines (2438 to $4072 \mathrm{~A}$ ) absorbed in his underwater spark spectrograms of iron. In our spectrograms a somewhat different experimental arrangement showed 263 iron lines absorbed. This number constitutes about 6 per cent of the total number of lines occurring in the arc emission spectrum of iron. The most prominent absorption lires involve the above-mentioned ${ }^{5} \mathrm{D}$ term, a considerable number of lines of 
moderate intensity involve the low metastable state ${ }^{5} \mathrm{~F}$, and a few partial reversals near the end of the table begin with the higher ${ }^{3} \mathrm{~F}$ term.

A few faint lines near $2400 \mathrm{~A}$ apparently originate with ionized atoms. The last are marked $\mathrm{Fe}^{+}$in Table 1. According to the analysis by Russell (21) these spark lines involve levels of the ${ }^{6} \mathrm{D}$ and ${ }^{4} \mathrm{D}$ terms, the former representing the normal state of the $\mathrm{Fe}^{+}$atom.

It is not altogether surprising that such fundamental lines should also be absorbed in a violent underwater spark, for the superposition of many bright spark lines on the continuous background indicates that some ionized atoms must be present, and our experience that even a small amount of an impurity in a metal can reveal absorption lines of the contaminating element resigns us to the belief that relatively few ionized atoms are required to produce absorption of the fundamental spark lines. Miss Stücklen had previously noted the appearance of the fundamental spark lines of $\mathrm{Cd}$ in underwater sparks, and we have frequently observed the $\mathrm{H}$ and $\mathrm{K}$ lines of $\mathrm{Ca}$ even when this element is present only as an impurity in other metallic electrodes.

The absorption data of the classified lines may be summarized briefly as follows: 120 lines the estimated intensities of which add up to 2,600 begin with the ${ }^{5} \mathrm{D}$ term, 76 lines with intensity. totaling 770 begin with the ${ }^{5} \mathrm{~F}$, and 11 lines with total intensity of 84 originate with ${ }^{3} \mathrm{~F}$. Among spark lines, 28 with total intensity 122 arise from ${ }^{6} \mathrm{D}$, 5 with intensity sum equal to 26 involve ${ }^{4} \mathrm{D}$, but no lines from the terms ${ }^{4} \mathrm{~F}$ and ${ }^{4} \mathrm{P}$ were observed in absorption, although ${ }^{4} \mathrm{~F}$ is much lower than ${ }^{4} \mathrm{D}$. There is apparently a connection between the lines observed in absorption and the electron configuration describing the initial energy states.

The value of underwater spark absorption spectra as an aid to the structural analysis of complex spectra is very well illustrated by the above data on iron. Out of a bewildering maze of 4,000 lines the underwater spark selects slightly more than 200 which are readily absorbed, and over half of these have one spectral term, namely that describing the normal state of the atom, in common.

$$
40735^{\circ}-27-2
$$


TABLE 1.-Underwater spark absorption spectrum of iron

\begin{tabular}{|c|c|c|c|c|c|c|c|c|c|c|c|}
\hline \multirow{2}{*}{$\lambda$} & \multirow{2}{*}{$\nu$} & \multicolumn{2}{|c|}{$\begin{array}{l}\text { Intensities } \\
\text { and class }\end{array}$} & \multirow{2}{*}{$\begin{array}{c}\text { Combina- } \\
\text { tion }\end{array}$} & \multirow{2}{*}{ Notes } & \multirow{2}{*}{$\lambda$} & \multirow{2}{*}{$\nu$} & \multicolumn{2}{|c|}{$\begin{array}{l}\text { Intensities } \\
\text { and class }\end{array}$} & \multirow{2}{*}{$\underset{\text { tion }}{\text { Combina- }}$} & \multirow{2}{*}{ Notes } \\
\hline & & A & $\mathrm{E}$ & & & & & A & $\mathbf{E}$ & & \\
\hline 2166.60 & 46140.7 & & $2 u$ & $a^{5} \mathrm{D}_{4}-d^{5} \mathrm{P}_{3} *$ & $\mathrm{~A}$ & 2410.526 & 41472.20 & 6 & 6 & $a^{6} \mathrm{D}_{2}-a^{6} \mathrm{~F}_{3}$ & $\mathrm{Fe}^{+}$ \\
\hline 66.79 & 136.6 & 100 & $2 \mathrm{u}$ & & & 11. 071 & 462.73 & 5 & 6 & $a^{6} \mathrm{D}_{1}-a^{6} \mathrm{~F}_{1}$ & $\mathrm{Fe}^{+}$ \\
\hline 71.20 & 46043.0 & 5 & 4 & & & 13.313 & 41424.21 & 4 & 6 & $a^{6} \mathrm{D}_{1}-a^{6} \mathrm{~F}_{2}$ & $\mathrm{Fe}^{+}$ \\
\hline 78.02 & 45898.8 & 100 & $4 \mathrm{u}$ & $a^{5} \mathrm{D}_{3}-d^{5} \mathrm{P}_{2} *$ & & 39.746 & 40975.45 & 3 & 4 & & \\
\hline 86.43 & 722.3 & & 3 & $a^{5} \mathrm{D}_{3}-d^{5} \mathrm{P}_{3} *$ & $\mathbf{A}$ & 57.602 & 677.75 & 6 & $6, \mathrm{II}$ & & \\
\hline 86.81 & 714.4 & 80 & 2 & & & 62.652 & 594.35 & 20 & $10 \mathrm{r}, \mathrm{II}$ & $a^{5} \mathrm{D}_{4}-c^{5} \mathrm{~F}_{4}$ & A \\
\hline 87.11 & 708.1 & & 4 & & & 65.155 & 553.14 & $2 ?$ & $6, \mathrm{III}$ & & \\
\hline 91.78 & 610.7 & 50 & $3 \mathrm{u}$ & $a^{5} \mathrm{D}_{2}-d^{5} \mathrm{P}_{2} *$ & A & 67.74 & 510.7 & 4 & 2 & $a^{5} \bar{F}_{3}-12_{2}^{*}$ & \\
\hline 2195.99 & 523.3 & 30 & 4 & $a^{5} \mathrm{D}_{1}-d^{5} \mathrm{P}_{1} *$ & & 68.885 & 491.86 & 5 & $4, \mathrm{III}$ & & \\
\hline 2200.34 & 433.3 & & 3 & $a^{5} D_{2}-d^{5} P_{3} *$ & & 72.875 & 426.54 & & 5 & $a^{5} \mathrm{D}_{2}-c^{5} \mathrm{~F}_{1}$ & \\
\hline & & 40 & & & - & & & 20 & $12 \mathrm{R}$ II & $a^{5} \mathrm{D}_{3}-c^{5} \mathrm{~F}$ & A \\
\hline 00.69 & 45426.1 & & 3 & $a^{5} \mathrm{D}_{0}-d^{5} \mathrm{P}_{1} *$ & & $\begin{array}{l}72.910 \\
79.782\end{array}$ & $\begin{array}{l}425.97 \\
313.95\end{array}$ & 10 & $\left|\begin{array}{cc}12 & R, I 1 \\
20 & R, I I\end{array}\right|$ & $\begin{array}{l}a^{5} \mathrm{D}_{3}-c^{6} \mathrm{~F}_{3} \\
a^{5} \mathrm{D}_{2}-c^{5} \mathrm{~F}_{2}\end{array}$ & \\
\hline 50.79 & 44415.0 & 2 & 2 & $a^{5} \mathrm{D}_{4}-1_{4}^{*}$ & & $\begin{array}{l}79.782 \\
83.277\end{array}$ & 257.21 & 50 & $60 \mathrm{R}, \mathrm{II}$ & $a^{5} \mathrm{D}_{4}-c^{5} \mathrm{~F}_{6}$ & $\begin{array}{l}\text { A } \\
\text { A }\end{array}$ \\
\hline 59.52 & 243.4 & 6 & 4 & $a^{5} \mathrm{D}_{4}-95^{*}$ & & $\begin{array}{l}83.277 \\
88.148\end{array}$ & 178.39 & 40 & $40 \mathrm{R}, \mathrm{II}$ & $a^{5} \mathrm{D}_{3}-c^{5} \mathrm{~F}_{4}$ & A \\
\hline 67.08 & 44095.9 & 3 & 2 & $a^{8} \mathrm{D}_{3}-d^{5} \mathrm{P}_{2} *$ & & $\begin{array}{l}88.148 \\
90.659\end{array}$ & 137.91) & & $30 \mathrm{R}, \mathrm{II}$ & $a^{5} \mathrm{D}_{2}-c^{5} \mathrm{~F}_{3}$ & A \\
\hline 72.04 & 43999.7 & 1 & 3. & $a^{5} \mathrm{D}_{3}-1_{4}^{*}$ & & & & 50 & & & A \\
\hline 75.98 & 923.5 & 4 & 3 & $a^{5} \mathrm{D}_{4}-d^{5} \overline{\mathrm{D}}_{3^{*}}$ & & 91.162 & 129.80 & & $20 \mathrm{R}, \mathrm{II}$ & $a^{5} \mathrm{D}_{1}-c^{5} \mathrm{~F}_{2}$ & A \\
\hline 84.06 & 768.1 & 3 & 5 & $a^{5} \mathrm{D}_{3}-d^{5} \overline{\mathrm{D}}_{2}{ }^{*}$ & A & 2496.539 & 40043.37 & $5 ?$ & $6, \mathrm{III}$ & & \\
\hline 87.27 & 706.7 & 3 & 3 & $a^{5} \mathrm{D}_{2}-a^{5} \overline{\mathrm{D}}_{1} *$ & & 2501.135 & 39969.80 & 20 & $20 \mathrm{R}, \mathrm{II}$ & $a^{5} \mathrm{D}_{4}-c^{6} \overline{\mathrm{D}_{3}}$ & A \\
\hline 94.42 & 570.5 & 1] & 2 & $a^{5} \mathrm{D}_{1}-d^{5} \overline{\mathrm{D}}_{0}^{*}$ & & 07.904 & 861.93 & 3 & $6, \mathrm{III}$ & & \\
\hline 94.69 & 565.4 & 1 & $5 ?$ & & & 10.837 & 815.36 & 20 & $15 \mathrm{R}, \mathrm{II}$ & $a^{5} \mathrm{D}_{3}-c^{6} \overline{\mathrm{D}}_{2}$ & $\mathbf{A}$ \\
\hline 2298.18 & 499.3 & 20 & $10 \mathrm{r}, \mathrm{II}$ & $a^{5} \mathrm{D}_{4}-a^{5} \overline{\mathrm{D}}_{4}^{*}$ & A & 12.366 & 791.14 & 5 & $5 \mathrm{r}, \mathrm{III}$ & & \\
\hline 2301. 66 & 433.5 & $1 ?$ & 5 & $a^{5} \mathrm{D}_{0}-d^{5} \overline{\mathrm{D}}_{1}^{*}$ & & 18. 107 & 700.43 & 25 & $12 \mathrm{r}, \mathrm{II}$ & $a^{5} \mathrm{D}_{2}-c^{5} \overline{\mathrm{D}}_{1}$ & A \\
\hline 03.43 & 400.1 & 3 & 2 & & & 22.855 & 625.71 & 40 & $40 \mathrm{R}, \mathrm{II}$ & $a^{5} \mathrm{D}_{4}-c^{5} \overline{\mathrm{D}}_{4}$ & A \\
\hline 03.56 & 397.7 & & $3, \mathrm{II}$ & & & 23.661 & 613.06 & 10 & & & \\
\hline 08.99 & 295.6 & 5 & $2, \mathrm{III}$ & $a^{5} \mathrm{D}_{1}-d^{6} \overline{\mathrm{D}}_{2}{ }^{*}$ & $A$ & 24.291 & 603.17 & 15 & $8 \mathrm{r}, \mathrm{II}$ & $a^{5} D_{1}-c^{5} \bar{D}_{0}$ & A \\
\hline 13.07 & 219.3 & 10 & $2, \mathrm{III}$ & $a^{5} \mathrm{D}_{2}-d^{5} \overline{\mathrm{D}}_{3} *$ & $A$ & 27.44 & 553.8 & 25 & $15 \mathrm{r}, \mathrm{II}$ & $a^{5} \mathrm{D}_{3}-c^{5} \overline{\mathrm{D}}_{3}$ & A \\
\hline 20.33 & 43084.1 & 20 & $2, \mathrm{III}$ & $a^{5} \mathrm{D}_{3}-d^{5} \overline{\mathrm{D}}_{3}{ }^{*}$ & $\mathbf{A}$ & 29.137 & 527.30 & 15 & $10 \mathrm{r}, \mathrm{II}$ & $a^{5} \mathrm{D}_{2}-c^{5} \overline{\mathrm{D}}_{2}$ & A \\
\hline 32.74 & 42854.9 & 5 & 6 & $a^{6} \mathrm{D}_{4}-a^{6} \mathrm{P}_{3}$ & $\mathrm{Fe}^{+}$ & 29.832 & 516.44 & 5 & 3, III & $a^{5} \mathrm{D}_{1}-c^{5} \overline{\mathrm{D}}_{1}$ & A \\
\hline 37.99 & 758.6 & 3 & 6 & $a^{6} \mathrm{D}_{2}-a^{6} \mathrm{P}_{2}$ & $\mathrm{Fe}^{+}$ & 35.610 & 426.40 & 15 & $8 \mathrm{r}, \mathrm{III}$ & $a^{5} \mathrm{D}_{0}-c^{5} \overline{\mathrm{D}}_{1}$ & $A$ \\
\hline 43.50 & 658.1 & 10 & 7 & $a^{6} \mathrm{D}_{5}-a^{6} \mathrm{P}_{4}$ & $\mathrm{Fe}^{+}$ & 37.180 & 402.00 & 10 & & & \\
\hline 44. 31 & 643.4 & 2 & 4 & $a^{6} \mathrm{D}_{1}-a^{6} \mathrm{P}_{2}$ & $\mathrm{Fe}^{+}$ & 40.976 & 343. 14 & 20 & $10 \mathrm{R}, \mathrm{III}$ & $a^{5} \mathrm{D}_{1}-c^{5} \overline{\mathrm{D}}_{2}$ & A \\
\hline 48.14 & 573.8 & 8 & 5 & & & 42.105 & 325.67 & 10 & $6, I V$ & & \\
\hline 48.32 & 570.6 & 8 & 5 & $a^{6} \mathrm{D}_{3}-a^{6} \mathrm{P}_{3}$ & $\mathrm{Fe}^{+}$ & 43.927 & 297.50 & 10 & $6, \mathrm{IV}$ & & \\
\hline 59.12 & 375.7 & 1 & 6 & $\left\{\begin{array}{l}a^{5} \mathrm{D}_{2}-c^{5} \mathrm{P}_{1} \\
?\end{array}\right.$ & & 44.716 & 285.32 & 5 & $6, I V$ & & \\
\hline 59.97 & 360.4 & 1 & 4 & $\left(a^{6} \mathrm{D}_{2}-a^{8} \mathrm{P}_{3}\right.$ & $\mathrm{Fe}^{+}$ & 45.979 & 265.83 & 20 & $10 \mathrm{r}, \mathrm{III}$ & $a^{5} \mathrm{D}_{2}-c^{5} \overline{\mathrm{D}}_{3}$ & A \\
\hline 60.31 & 354.4 & 1 & $5 \mathrm{u}$ & & & 49. 616 & 209.82 & 15 & $10 \mathrm{r}, \mathrm{III}$ & $a^{5} \mathrm{D}_{3}-c^{5} \overline{\mathrm{D}}_{4}$ & A \\
\hline 64.82 & 273.6 & 5 & 8 & $a^{6} \mathrm{D}_{4}-a^{6} \mathrm{P}_{4}$ & $\mathrm{Fe}^{+}$ & 62.543 & 39012.05 & 1 & 5 & & $\mathrm{Fe}^{+}$ \\
\hline 69.47 & 190.6 & 2 & 2 & $a^{5} \mathrm{D}_{1}-c^{5} \mathrm{P}_{1}$ & & 76. 699 & 38797.73 & 5 & 4, III & $a^{5} \overline{\mathrm{F}}_{5}-c^{5} \mathrm{G}_{b} *$ & \\
\hline 71.44 & 155.6 & 6 & 4 & $a^{5} \mathrm{D}_{2}-c^{5} \mathrm{P}_{2}$ & & 84.544 & 679. 98 & 10 & 8, III & $a^{5} \overline{\mathrm{F}}_{5}-c^{5} \overline{\mathrm{G}}_{6} *$ & \\
\hline 73. 624 & 116.80 & 10 & 4 & $a^{5} \mathrm{D}_{3}-c^{5} \mathrm{P}_{3}$ & & 85.886 & 659.90 & 5 & $20, I V$ & $a^{6} \mathrm{D}_{5}-a^{6} \overline{\mathrm{D}}_{4}$ & $\mathrm{Fe}^{+}$ \\
\hline 73. 733 & 114.87 & 10 & $15, \mathrm{II}$ & $a^{6} \mathrm{D}_{5}-a^{6} \mathrm{~F}_{5}$ & $\mathrm{Fe}^{+}$ & 98.380 & 474,03 & 6 & 7 & $a_{6} \mathrm{D}_{4}-a_{6} \overline{\mathrm{D}}_{3}$ & $\mathrm{Fe}^{+}$ \\
\hline 74.54 & 42100.6 & 2 & $?$ & & & 2599.405 & 458.86 & 15 & $6 r$ & $a^{6} \mathrm{D}_{5}-a^{6} \overline{\mathrm{D}}_{5}$ & $\mathrm{Fe}^{+}$ \\
\hline 80.763 & 41990.53 & 1 & 4 & $a^{6} \mathrm{D}_{3}-a^{6} \mathrm{P}_{4}$ & $\mathrm{Fe}^{+}$ & 2605. 687 & 366.14 & 2 & $6, \mathrm{III}$ & & \\
\hline 81.85 & 971.4 & 4 & 2 & $a^{5} \mathrm{D}_{1}-c^{5} \mathrm{P}_{2}$ & & 06.839 & 349.18 & 6 & $6, \mathrm{III}$ & $a^{5} \mathrm{~F}_{4}-c^{6} \overline{\mathrm{G}}_{5} *$ & \\
\hline 82.039 & 968.03 & 10 & $20, \mathrm{II}$ & $a^{5} \mathrm{D}_{5}-a^{6} \mathrm{~F}_{6}$ & $\mathrm{Fe}^{+}$ & 07.099 & 345.37 & 4 & 7 & $a^{6} \mathrm{D}_{3}-a^{6} \overline{\mathrm{D}}_{3}$ & $\mathrm{Fe}^{+}$ \\
\hline 88.631 & 852.22 & 7 & 6 & $a^{5} \mathrm{D}_{4}-a^{6} \mathrm{~F}_{4}$ & $\mathrm{Fe}^{+}$ & 11.885 & 275.10 & 5 & 8 & $a_{6} \mathrm{D}_{4}-a_{6} \overline{\mathrm{D}}_{4}$ & $\mathrm{Fe}^{+}$ \\
\hline 89.979 & 828.60 & 8 & 4 & $a^{5} \mathrm{D}_{2}-c^{5} \mathrm{P}_{3}$ & & 13. 835 & 246.55 & 2 & 8 & $a_{6} \mathrm{D}_{2}-a_{6} \overline{\mathrm{D}}_{1}$ & $\mathrm{Fe}^{+}$ \\
\hline 95.628 & 729.99 & 8 & 8 & $a^{6} \mathrm{D}_{4}-a^{6} \mathrm{~F}_{5}$ & $\mathrm{Fe}^{+}$ & 17.627 & 191.15 & 1 & 6 & $a^{6} \mathrm{D}_{3}-a^{6} \overline{\mathrm{D}}_{3}$ & $\mathrm{Fe}^{+}$ \\
\hline 2399. 244 & 667.10 & 5 & 6 & $a^{6} \mathrm{D}_{3}-a^{6} \mathrm{~F}_{3}$ & $\mathrm{Fe}^{+}$ & 18. 027 & 185.32 & 4 & $5, \mathrm{III}$ & $a^{5} \overline{\mathrm{F}}_{3}-a_{6}^{5} \overline{\mathrm{G}}_{3} *$ & \\
\hline 2404.888 & 41569.32 & 5 & 6 & $a^{6} \mathrm{D}_{3}-a^{6} \mathrm{~F}_{4}$ & $\mathrm{Fe}^{+}$ & 2623. $544 \mid 3$ & 38105.02 & 6 & $5, \mathrm{III}$ & $a^{6} \overline{\mathrm{F}}_{3}-c^{5} \overline{\mathrm{G}}_{4} *$ & \\
\hline
\end{tabular}


TABLE 1.-Underwater spark absorption spectrum of iron-Continued

\begin{tabular}{|c|c|c|c|c|c|c|c|c|c|c|c|}
\hline \multirow{2}{*}{$\lambda$} & \multirow{2}{*}{$\nu$} & \multicolumn{2}{|c|}{$\begin{array}{l}\text { Intensities } \\
\text { and class }\end{array}$} & \multirow{2}{*}{$\begin{array}{c}\text { Combina- } \\
\text { tion }\end{array}$} & \multirow{2}{*}{ Notes } & \multirow{2}{*}{$\boldsymbol{\lambda}$} & \multirow{2}{*}{$\nu$} & \multicolumn{2}{|c|}{$\begin{array}{l}\text { Intensities } \\
\text { and class }\end{array}$} & \multirow{2}{*}{$\begin{array}{c}\text { Combina- } \\
\text { tion }\end{array}$} & \multirow{2}{*}{ Notes } \\
\hline & & A & $\mathbf{E}$ & & & & & A & $\mathrm{E}$ & & \\
\hline 2625.676 & 38074.09 & 3 & 8 & $a^{6} \mathrm{D}_{4}-a^{6} \overline{\mathrm{D}}_{5}$ & $\mathrm{Fe}^{+}$ & 2804.523 & 35646.20 & 4 & $20, \mathrm{II}$ & $a^{5} \bar{F}_{4}-b^{5} \overline{\mathrm{G}}_{4}$ & \\
\hline 28.303 & 38036.03 & 2 & 6 & $a^{6} \mathrm{D}_{1}-a^{0} \overline{\mathrm{D}}_{2}$ & $\mathrm{Fe}^{+}$ & 06.985 & 614.94 & 4 & $20, \mathrm{II}$ & $a^{5} \overline{\mathrm{F}}_{4}-2{ }_{4}^{*}$ & \\
\hline 31.053 & 37996.27 & 5 & 6 & $a^{6} \mathrm{D}_{2}-a^{6} \overline{\mathrm{D}}_{3}$ & $\mathrm{Fe}^{+}$ & 13. 288 & 535.15 & 10 & $30 \mathrm{R}, \mathrm{II}$ & $a^{5} \overline{\mathrm{F}}_{4}-b^{6} \overline{\mathrm{G}}_{5}$ & \\
\hline 31.332 & 992.25 & 5 & & $a^{6} \mathrm{D}_{3}-a^{6} \overline{\mathrm{D}}_{4}$ & $\mathrm{Fe}^{+}$ & 23.276 & 409.45 & 2 & $20, \mathrm{II}$ & $a^{5} \overline{\mathrm{F}}_{3}-b^{5} \overline{\mathrm{G}}_{5}$ & \\
\hline 32.248 & 979.03 & 2 & 4, III & $a^{5} \overline{\mathrm{F}}_{2}-\mathrm{c}^{5} \overline{\mathrm{G}}_{2}{ }^{*}$ & & 25.556 & 380.86 & & $20, \mathrm{II}$ & $a^{5} \overline{\mathrm{F}}_{3}-b^{5} \overline{\mathrm{G}}_{3}$ & \\
\hline 35.818 & 927.59 & 5 & $8, \mathrm{III}$ & $a^{5} \overline{\mathrm{F}}_{2}-c^{5} \overline{\mathrm{G}}_{3} *$ & & 25.687 & 379.23 & & $6, \mathrm{II}$ & $a^{5} \mathrm{D}_{4}-a^{3} \overline{\mathrm{G}}_{5}$ & \\
\hline 41.654 & 843.81 & 1 & 4, IIII & & & 32.433 & 294.96 & 10 & $25 \mathrm{r}, \mathrm{II}$ & $a^{5} \overline{\mathrm{F}}_{3}-b^{5} \overline{\mathrm{G}}_{4}$ & \\
\hline 44.008 & 810.12 & 5 & $8, \mathrm{III}$ & $a^{5} \overline{\mathrm{F}}_{1}-c^{5} \overline{\mathrm{G}}_{2}{ }^{*}$ & & 38.118 & 224.28 & 2 & $10, \mathrm{III}$ & $a^{5} \overline{\mathrm{F}}_{2}-b^{5} \overline{\mathrm{G}}_{2}$ & \\
\hline 66.818 & 486.74\} & 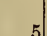 & $8, \mathrm{III}$ & $a^{6} \overline{\mathrm{F}}_{5}-1_{4}{ }^{*}$ & & 43.629 & 156.02 & 6 & $10, \mathrm{III}$ & $a^{5} \overline{\mathrm{F}}_{4}-c^{5} \mathrm{P}_{3}$ & \\
\hline 66.970 & 484. 62$\}$ & & $3, \mathrm{III}$ & & & 43.974 & 151.76 & 8 & $20 \mathrm{r}, \mathrm{IJ}$ & $a^{5} \overline{\mathrm{F}_{2}}-b^{5} \overline{\mathrm{G}}_{3}$ & \\
\hline 79.066 & 315.37 & 10 & $10, \mathrm{III}$ & $a^{5} \overline{\bar{F}^{5}}-9 b^{*}$ & & 51.7983 & 35055.32 & 10 & $15 \mathrm{r}, \mathrm{II}$ & $a^{5} \overline{\mathrm{F}}_{1}-b^{5} \overline{\mathrm{G}_{8}}$ & \\
\hline 89.220 & 174.48 & 5 & $8, \mathrm{III}$ & $a^{5} \bar{F}_{4}-8_{3}^{*}$ & & $\begin{array}{l}51.798 \\
63.866\end{array}$ & 34907.60 & 1 & $8, \mathrm{I}$ & $a^{5} \mathrm{D}_{2}-a^{5} \overline{\mathrm{G}_{3}}$ & \\
\hline 2699.114: & 7038.22 & 2 & $6, \mathrm{III}$ & 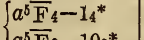 & & 69.313 & 841.34 & 2 & $10, \mathrm{I}$ & $a^{5} \mathrm{D}_{3}-a^{5} \overline{\mathrm{G}}_{4}$ & \\
\hline & & 2 & & $\mid a^{5} \bar{F}_{2}-102^{*}$ & & 2874.176 & 782.39 & 5 & $10, \mathrm{I}$ & $a^{5} \mathrm{D}_{4}-a^{5} \overline{\mathrm{G}}_{5}$ & \\
\hline $\begin{array}{r}2706.590 \\
11.662\end{array}$ & 66935. 91 & 4 & 8, III & & & 2912.161 & 328.72 & 3 & $20 \mathrm{r}, \mathrm{I}$ & $a^{5} \mathrm{D}_{4}-b^{5} \mathrm{~F}_{8}$ & \\
\hline & & & & & & 29.006 & 131.31 & 5 & $25 \mathrm{r}, \mathrm{I}$ & $a^{5} \mathrm{D}_{3}-b^{5} \mathrm{~F}_{2}$ & \\
\hline $\begin{array}{l}18.445 \\
19.037\end{array}$ & $\begin{array}{l}774.86 \\
766.85\end{array}$ & 10 & 6, III & $a^{5} \overline{\mathrm{F}}_{2}-7_{1}{ }^{*}$ & & 36.903 & 34039.54 & 20 & $60 \mathrm{R}, \mathrm{I}$ & $a^{5} \mathrm{D}_{4}-b^{5} \mathrm{~F}_{4}$ & A \\
\hline $\begin{array}{l}19.037 \\
20.910\end{array}$ & $\begin{array}{l}766.85 \\
741.54\end{array}$ & $\begin{array}{l}50 \\
40\end{array}$ & $60 \mathrm{R}, \mathrm{II}$ & $a^{5} \mathrm{D}_{4}-b^{5} \mathrm{P}_{3}$ & $A, C t$ & 41.343 & 33988.16 & 2 & $15 \mathrm{r}, \mathrm{I}$ & $a^{5} \mathrm{D}_{2}-b^{5} \mathrm{~F}_{1}$ & \\
\hline $\begin{array}{l}20.910 \\
23.582\end{array}$ & $\begin{array}{l}71.54 \\
705.50\end{array}$ & $\begin{array}{l}40 \\
25\end{array}$ & $\begin{array}{r}40 \mathrm{r}, \mathrm{II} \\
15, \mathrm{II}\end{array}$ & $\begin{array}{l}a^{5} \mathrm{D}_{3}-b^{6} \mathrm{P}_{2} \\
a^{5} \mathrm{D}_{2}-b^{5} \mathrm{P}_{1}\end{array}$ & $\mathrm{~A}$ & 47.876 & 912.84 & 25 & $60 \mathrm{R}, \mathrm{I}$ & $a^{5} \mathrm{D}_{3}-b^{3} \mathrm{~F}_{3}$ & A \\
\hline 26.064 & 672.09 & 10 & $6, \mathrm{III}$ & $a^{5} \overline{\mathrm{F}}_{5}-4_{4}^{*}$ & & 53. 943 & 843.18 & 20 & $50 \mathrm{R}, \mathrm{II}$ & $a^{5} \mathrm{D}_{2}-b^{5} \mathrm{~F}_{2}$ & A \\
\hline 28.026 & 645.71 & 3 & $3, \mathrm{III}$ & $a^{5} \overline{\mathrm{F}}_{4}-5_{4} *$ & - & 57.370 & 803.98 & 10 & $30 \mathrm{R}, \mathrm{II}$ & $a^{5} \mathrm{D}_{1}-b^{5} \mathrm{~F}_{1}$ & \\
\hline 33.580 & 571.26 & 15 & $15, \mathrm{II}$ & $a^{5} \overline{\mathrm{F}}_{5}-d^{5} \overline{\mathrm{D}}_{4}^{*}$ & & 65.258 & 714.06 & 8 & 20, II & $a^{5} \mathrm{D}_{0}-b^{5} \mathrm{~F}_{1}$ & \\
\hline 35.480 & 545.86 & 10 & 8, III & $a^{5} \overline{\mathrm{F}}_{4}-d^{5} \overline{\mathrm{D}}_{3} *$ & & 66.902 & 695.38 & 50 & $125 \mathrm{R}, \mathrm{II}$ & $a^{5} \mathrm{D}_{4}-b^{5} \mathrm{~F}_{5}$ & $A, G$ \\
\hline 37.312 & 521.40 & 25 & $20 \mathrm{r}, \mathrm{II}$ & $a^{5} \mathrm{D}_{1}-b^{5} \mathrm{P}_{1}$ & & 69.364 & $667.43\}$ & ( & $5, \mathrm{II}$ & $a^{5} \mathrm{D}_{1}-a^{3} \mathrm{P}_{0}$ & \\
\hline 39.551 & 491.55 & 5 & & $a^{4} \mathrm{D}_{4}-a^{4} \overline{\mathrm{D}}_{4}$ & $\mathrm{Fe}^{+}$ & 69.482 & $666.09\}$ & 2 & $10, I$ & $a^{5} \overline{\mathrm{F}}_{5}-c^{5} \mathrm{~F}_{4}$ & \\
\hline 42.408 & 453.53 & 30 & $30 \mathrm{r}, \mathrm{II}$ & $a^{5} \mathrm{D}_{2}-b^{5} \mathrm{P}_{2}$ & A & & & & & $\int a^{5} \mathrm{D}_{1}-b^{5} \mathrm{~F}_{2}$ & A \\
\hline 43.566 & 438.15 & 1 & $3, \mathrm{III}$ & $a^{5} \overline{\mathrm{F}}_{4}-32^{*}$ & & 70.107 & 659.02 & 10 & $40 \mathrm{R}, \mathrm{I}$ & $\left\{\begin{array}{l}a^{5} \mathrm{D}_{2}-a^{3} \mathrm{P}_{1}\end{array}\right.$ & $\Omega$ \\
\hline 44.072 & 431.44 & 20 & $10, \mathrm{II}$ & $a^{5} \underline{\mathrm{D}}_{0}-b^{5} \underline{\mathrm{P}}_{1}$ & & 73.137 & $624.72\}$ & & $60 \mathrm{R}, \mathrm{I}$ & $a^{5} \mathrm{D}_{2}-b^{5} \mathrm{~F}_{3}$ & \\
\hline 44. 531 & 425.34 & 5 & 8, IIII & $a^{6} \overline{\mathrm{F}}_{2}-d^{5} \overline{\mathrm{D}}_{1} *$ & & 73.236 & $623.60\}$ & 50 & $60 \mathrm{R}, \mathrm{I}$ & $a^{5} \mathrm{D}_{3}-b^{5} \mathrm{~F}_{4}$ & $G\}^{A}$ \\
\hline 46.486 & 399.42 & 4 & 7 & $a^{4} \mathrm{D}_{2}-a^{4} \mathrm{~F}_{3}$ & $\mathrm{Fe}^{+}$ & 81.448 & 531.00 & 8 & $20 \mathrm{r}, \mathrm{I}$ & $a^{5} \mathrm{D}_{3}-a^{3} \mathrm{P}_{2}$ & \\
\hline 46.988 & 392.76 & 5 & $20, \mathrm{III}$ & $a^{4} \mathrm{D}_{3}-a^{4} \overline{\mathrm{D}}_{3}$ & $\mathrm{Fe}^{+}$ & 83.571 & 507.13 & 75 & $125 \mathrm{R}, \mathrm{I}$ & $a^{5} \mathrm{D}_{4}-b^{5} \overline{\mathrm{D}}_{3}$ & A \\
\hline 49. 324 & 361.84 & 8 & & $a^{4} \mathrm{D}_{3}-a^{4} \mathrm{~F}_{4}$ & $\mathrm{Fe}^{+}$ & & & & & & \\
\hline 50.145 & 351.00 & 25 & $25 \mathrm{r}, \mathrm{II}$ & $a^{5} \mathrm{D}_{3}-b^{5} \mathrm{P}_{3}$ & A & & & 2 & 10, III & $a^{5} \mathrm{~F}_{4}-c^{6} \mathrm{~F}_{3}$ & \\
\hline 53.688 & 304.22 & 2 & $3, \mathrm{III}$ & $\left\{a^{5} \overline{\mathrm{F}}_{2}-61^{*}\right.$ & & $\begin{array}{r}94.434 \\
2999.516\end{array}$ & $\begin{array}{l}385.58 \\
329.02\end{array}$ & $\begin{array}{r}50 \\
5\end{array}$ & $\left|\begin{array}{l}100 \mathrm{R}, \mathrm{I} \\
30 \mathrm{R}, \mathrm{II}\end{array}\right|$ & $\begin{array}{l}a^{5} \mathrm{D}_{3}-b^{5} \overline{\mathrm{D}}_{2} \\
a^{5} \overline{\mathrm{F}}_{5}-c^{5} \mathrm{~F}_{5}\end{array}$ & A \\
\hline 54.032 & 299.69 & 2 & $3, \mathrm{III}$ & $\left(a^{5} \overline{\mathrm{F}} 1-d^{5} \overline{\mathrm{D}} 0^{*}\right.$ & & 3000.951 & 313.09 & 40 & $100 \mathrm{R}, \mathrm{I}$ & $a^{5} \mathrm{D}_{2}-b^{5} \overline{\mathrm{D}}_{1}$ & A \\
\hline 55.736 & 277.24 & 4 & 8 & $a^{4} \mathrm{D}-a^{4} \mathrm{~F}_{5}$ & $\mathrm{Fe}^{+}$ & 07.284 & 242.94 & 4 & $12 \mathrm{r}, \mathrm{I}$ & $a^{5} \mathrm{D}_{2}-a^{3} \mathrm{P}_{2}$ & \\
\hline 56.332 & 269.40 & 10 & $20, \mathrm{I}$ & $a^{5} \mathrm{D}_{1}-b^{5} \mathrm{P}_{2}$ & & 08.142 & 233.46 & 20 & $60 \mathrm{R}, \mathrm{I}$ & $a^{5} \mathrm{D}_{1}-b^{5} \overline{\mathrm{D}_{0}}$ & \\
\hline 57.316 & 256.4 & 3 & $10, \mathrm{III}$ & $a^{5} \overline{\mathrm{F}} 1-d^{5} \overline{\mathrm{D}}_{1} *$ & & 09.575 & 217.63 & 4 & $25 \mathrm{r}, \mathrm{II}$ & $a^{5} \overline{\mathrm{F}}_{4}-c^{5} \bar{F}_{4}$ & \\
\hline 59.816 & 223.60 & $1 ?$ & $4, \mathrm{III}$ & & & 17.630 & 128.96 & 3 & $15 \mathrm{r}, \mathrm{I}, \mathrm{A}$ & $a^{5} \overline{\mathrm{D}}_{1}-b^{5} \overline{\mathrm{D}}_{1}$ & \\
\hline 61.788 & 197. 76 & 1 ? & $18, \mathrm{III}$ & $a^{5} \overline{\mathrm{F}}_{2}-d^{5} \overline{\mathrm{D}}_{2} *$ & & & & & & & \\
\hline 62.029 & 194.59 & 2 & $15, \mathrm{III}$ & $a^{5} \overline{\mathrm{F}}_{3}-d^{5} \overline{\mathrm{D}}_{3} *$ & & 20.495 & 097. 54 & 100 & $100 \mathrm{R}, \mathrm{II}$ & $a^{b} \mathrm{D}_{2}-b^{5} \overline{\mathrm{D}}_{2}$ & \\
\hline 63.107 & 180.48 & $1 ?$ & $4, \mathrm{III}$ & $a^{5} \bar{F} 3-32^{*}$ & & & $095.92 \mid \int$ & & $200 \mathrm{R}, \mathrm{I}$ & $a^{5} \mathrm{D}_{4}-b^{5} \overline{\mathrm{D}}_{4}$ & G \\
\hline 66.910 & 130.74 & $1 ?$ & $2, \mathrm{III}$ & & & 21.076 & 091.18 & 50 & $150 \mathrm{R}, \mathrm{I} \mid$ & $a^{5} \mathrm{D}_{3}-b^{5} \overline{\mathrm{D}}_{3}$ & G. \\
\hline 67.518 & 122.80 & 4 & $20, \mathrm{III}$ & $a^{5} \overline{\mathrm{F}}_{4}-d^{5} \overline{\mathrm{D}}_{4}^{*}$ & & 24.035 & 058.78 & 3 & $15 \mathrm{r}, \mathrm{I}, \mathrm{A}$ & $a^{5} \mathrm{D}_{1}-a^{3} \mathrm{P}_{2}$ & \\
\hline 72.083 & 063. 33 ] & & $20, \mathrm{II}$ & $a^{5} \overline{\mathrm{F}}_{5}-24^{*}$ & & 25.846 & 33039.02 & 10 & $50 \mathrm{R}, \mathrm{I}$ & $a^{5} \mathrm{D}_{0}-b^{5} \overline{\mathrm{D}}_{1}$ & \\
\hline 72.112 & 6062.95 & 10 & $1, \mathrm{III}$ & $a^{5} \mathrm{D}_{2}-b^{5} \mathrm{P}_{3}$ & & 37.392 & 32913.43 & 20 & $80 \mathrm{R}, \mathrm{I}$ & $a^{5} \mathrm{D}_{1}-b^{5} \overline{\mathrm{D}}_{2}$ & A \\
\hline 78.226 & 5983.60 & 5 & $20, \mathrm{III}$ & $a^{5} \overline{\mathrm{F}}_{5}-b^{5} \overline{\mathrm{G}}_{5}$ & & 47.608 & 803.10 & 300 & $100 \mathrm{R}, \mathrm{I}$ & $a^{5} \mathrm{D}_{2}-b^{5} \overline{\mathrm{D}_{3}}$ & $A, G$ \\
\hline 88.108 & 856.06 & 15 & $30, \mathrm{II}$ & $a^{5} \overline{\mathrm{F}}_{8}-b^{5} \overline{\mathrm{G}}_{8}$ & & 57.451 & 697.50 & 10 & $40 \mathrm{R}, \mathrm{II}$ & $a^{5} \overline{\mathrm{F}}_{8}-c^{5} \overline{\mathrm{D}}_{4}$ & \\
\hline 94.706 & $771.41\}$ & & 2 & $a^{5} \overline{\mathrm{F}}_{3}-d^{5} \overline{\mathrm{D}}_{4}^{*}$ & & 59.090 & 679.98 & 30 & $100 \mathrm{R}, \mathrm{I}$ & $a^{5} \bar{D}_{3}-b^{6} \overline{\mathrm{D}}_{4}$ & \\
\hline 2795.008 & $5767.55\}\}$ & & $3, \mathrm{III}$ & $a^{5} \mathrm{D}_{4}-a^{8} \overline{\mathrm{C}}_{4} \mid$ & & 3067.2503 & 32593.05 & 3 & $30 \mathrm{r}, \mathrm{II}$ & $a^{5} \overline{\mathrm{F}}_{\mathbf{q}}-c^{5} \overline{\mathrm{D}_{3}}$ & \\
\hline
\end{tabular}


TABLE 1.-Underwater spark absorption spectrum of iron-Continued

\begin{tabular}{|c|c|c|c|c|c|c|c|c|c|c|c|}
\hline \multirow{2}{*}{$\lambda$} & \multirow{2}{*}{$\nu$} & \multicolumn{2}{|c|}{$\begin{array}{l}\text { Intensities } \\
\text { and class }\end{array}$} & \multirow{2}{*}{$\underset{\text { tion }}{\text { Combina- }}$} & \multirow{2}{*}{ Notes } & \multirow{2}{*}{$\lambda$} & \multirow{2}{*}{$\nu$} & \multicolumn{2}{|c|}{$\begin{array}{l}\text { Intensities } \\
\text { and class }\end{array}$} & \multirow{2}{*}{$\underset{\text { tion }}{\text { Combina- }}$} & \multirow{2}{*}{ Notes } \\
\hline & & A & $\mathrm{E}$ & & & & & A & $\mathrm{E}$ & & \\
\hline 3075.725 & 32503.25 & 2 & $25 \mathrm{r}, \mathrm{II}$ & $a^{5} \overline{\mathrm{F}}_{3}-c^{5} \overline{\mathrm{D}}_{2}$ & & 3737.135 & 26750.88 & 60 & $150 \mathrm{R}, \mathrm{I}$ & $a^{5} \mathrm{D}_{3}-a^{5} \mathrm{~F}_{4}$ & $G$ \\
\hline 3083.745 & 32418.71 & 1 & $20, \mathrm{II}$ & $a^{5} \overline{\mathrm{F}}_{2}-c^{5} \overline{\mathrm{D}}_{1}$ & & 45.563 & 690.69 & 70 & $100 \mathrm{R}, \mathrm{I}$ & $a^{5} \mathrm{D}_{2}-a^{5} \mathrm{~F}_{3}$ & $\mathrm{~A}, \mathrm{G}$ \\
\hline 3236.231 & 30891.25 & $1 ?$ & 20 r,I,A & $a^{5} \mathrm{D}_{3}-0^{3} \mathrm{~F}_{4}$ & & 45.900 & 688.29 & 70 & $40 \mathrm{r}, \mathrm{I}, \mathrm{A}$ & $a^{5} \mathrm{D}_{0}-a^{5} \mathrm{~F}_{1}$ & \\
\hline 3440.614 & 29056.28 & 20 & $150 \mathrm{R}, \mathrm{I}$ & $a^{5} \mathrm{D}_{4}-a^{5} \mathrm{P}_{3}$ & $\mathbf{A}$ & 48.264 & 671.47 & & $60 \mathrm{R}, \mathrm{I}, \mathrm{A}$ & $a^{5} \mathrm{D}_{1}-a^{5} \mathrm{~F}_{2}$ & $\stackrel{A}{ }$ \\
\hline 40.992 & 053.09 & 10 & $75 \mathrm{R}, \mathrm{I}$ & $a^{5} \mathrm{D}_{3}-a^{5} \mathrm{P}_{2}$ & & 49. 487 & 662.76 & 30 & $200 \mathrm{R}, \mathrm{II} \mid$ & $a^{5} \overline{\mathbf{F}}_{4}-b^{5} \bar{F}_{4}$ & \\
\hline 43.883 & 29028.70 & 5 & $50 \mathrm{r}, \mathrm{I}$ & $a^{5} \mathrm{D}_{2}-a^{5} \mathrm{P}_{1}$ & & 58.234 & 600.71 & 20 & $150 \mathrm{R}, \mathrm{II}$ & $a^{5} \overline{\mathrm{F}}_{3}-b^{5} \mathrm{~F}_{3}$ & \\
\hline 65.864 & 28844.59 & 10 & $60 \mathrm{r}, \mathrm{I}$ & $a^{5} \mathrm{D}_{1}-a^{5} \mathrm{P}_{1}$ & & 63.792 & 561.43 & 10 & $100 \mathrm{r}, \mathrm{II}$ & $a^{5} \overline{\mathrm{F}}_{2}-b^{5} \mathrm{~F}_{2}$ & \\
\hline 75.454 & 765.00 & 15 & $70 \mathrm{r}, \mathrm{I}$ & $a^{5} \mathrm{D}_{2}-a^{5} \mathrm{P}_{2}$ & & 3767.194 & 537.44 & 8 & $80 \mathrm{r}, \mathrm{II}$ & $a^{5} \overline{\mathrm{F}}_{1}-b^{5} \mathrm{~F}_{1}$ & \\
\hline 76. 705 & 754.66 & 5 & $40, \mathrm{I}$ & $a^{5} \mathrm{D}_{0}-a^{5} \mathrm{P}_{1}$ & & 3812.966 & 218.89 & $1 ?$ & $40, \mathrm{II}$ & $a^{8} \overline{\mathrm{F}}_{3}-a^{3} \mathrm{P}_{2}$ & \\
\hline 90.577 & 640.40 & 25 & $100 \mathrm{r}, \mathrm{I}$ & $a^{5} \mathrm{D}_{3}-a^{5} \mathrm{P}_{3}$ & & 15.844 & 199.60 & 10 & $100 \mathrm{r}, \mathrm{II}$ & $a^{3} \overline{\mathrm{F}}_{4}-a^{3} \overline{\mathrm{D}_{3}}$ & \\
\hline 3497.842 & 580.90 & 5 & $40, \mathrm{I}$ & $a^{5} \mathrm{D}_{1}-a^{5} \mathrm{P}_{2}$ & & 20.430 & 167.67 & 30 & $250 \mathrm{R}, \mathrm{II}$ & $a^{5} \overline{\mathrm{F}_{8}}-b^{5} \overline{\mathrm{D}}_{4}$ & \\
\hline 3513.821 & 450.93 & 1 & $30, \mathrm{II}$ & $a^{5} \overline{\mathrm{F}}_{5}-a^{3} \overline{\mathrm{G}}_{5}$ & & 24.444 & 140.20 & 10 & $50 \mathrm{r}, \mathrm{I}, \mathrm{A}$ & $a^{8} \mathrm{D}_{4}-a^{5} \overline{\mathrm{D}}_{3}$ & \\
\hline 21.264 & 390.80 & 1 & $25, \mathrm{II}$ & $a^{5} \overline{\mathrm{F}}_{4}-a^{3} \overline{\mathrm{G}}_{4}$ & & 25.886 & 130.35 & 20 & $200 \mathrm{R}, \mathrm{II}$ & $a^{5} \overline{\mathrm{F}} 5-b^{5} \overline{\mathrm{D}} 3$ & \\
\hline 26.069 & 352.11 & & $20, \mathrm{I}$ & $a^{5} \underline{D}_{2}-a^{5} P_{3}$ & & 27.826 & 117.10 & 10 & $75 \mathrm{r}, \mathrm{II}$ & $a^{3} \overline{\mathrm{F}}_{3}-a^{3} \overline{\mathrm{D}}_{2}$ & \\
\hline 26.167 & .351 .34 & 5 & $15, \mathrm{II}$ & $a^{5} \overline{\mathrm{F}}_{3}-a^{3} \overline{\mathrm{G}}_{3}$ & & 34.227 & 073.51 & 10 & $100 \mathrm{r}, \mathrm{II}$ & $a^{5} \overline{\mathrm{F}}_{3}-b^{6} \overline{\mathrm{D}}_{2}$ & \\
\hline 58.522 & 093.55 & 5 & $30, \mathrm{II}$ & $a^{5} \overline{\overline{\mathrm{F}}}_{2}-a^{3} \overline{\mathrm{G}}_{3}$ & & 40.443 & 031.30 & 5 & $80 \mathrm{r}, \mathrm{II}$ & $a^{6} \overline{\mathrm{F}_{2}}-b^{5} \overline{\mathrm{D} 1}$ & \\
\hline 65.383 & 039.50 & 20 & $60 \mathrm{r}, \mathrm{II}$ & $a^{5} \overline{\mathrm{F}}_{3}-a^{3} \overline{\mathrm{G}}_{4}$ & & 41.052 & 26027.18 & 5 & $80 \mathrm{r}, \mathrm{II}$ & $a^{3} \overline{\mathrm{F}}_{2}-a^{3} \overline{\bar{D}}_{1}$ & \\
\hline 70.102 & 28002.44 & 40 & $100 \mathrm{R}, \mathrm{I}$ & $a^{5} \overline{\mathrm{F}}_{4}-a^{3} \overline{\mathrm{G}}_{8}$ & & 56.373 & 25923.78 & 5 & $50 \mathrm{r}, \mathrm{I}, \mathrm{A}$ & $a^{5} \mathrm{D}_{3}-a^{5} \overline{\mathrm{D}}_{2}$ & \\
\hline 81.197 & 27915.68 & 100 & $250 \mathrm{R}, \mathrm{I}$ & $a^{5} \overline{\mathrm{F}}_{5}-a^{5} \overline{\mathrm{G}}_{6}$ & & 59.913 & 900.00 & 50 & $300 \mathrm{R}, \mathrm{I}$ & $a^{5} \mathrm{D}_{4}-a^{5} \overline{\mathrm{D}}_{4}$ & $\mathrm{~A}, \mathrm{G}$ \\
\hline 85.322 & 883.56 & 4 & $30, \mathrm{II}$ & $a^{5} \overline{\mathrm{F}}_{3}-a^{5} \overline{\mathrm{G}}_{3}$ & & 78.578 & 775.38 & 5 & $100 \mathrm{r}, \mathrm{II}$ & $a^{5} \mathrm{D}_{2}-a^{5} \overline{\mathrm{D}}_{1}$ & \\
\hline 85.708 & 880.57 & 3 & $20, \mathrm{II}$ & $a^{5} \overline{\mathrm{F}}_{4}-a^{5} \overline{\mathrm{G}}_{4}$ & & 86. 287 & 724.24 & 30 & $40 \mathrm{R}, \mathrm{I}$ & $a^{5} \mathrm{D}_{3}-a^{5} \overline{\mathrm{D}}_{3}$ & $G$ \\
\hline 3586.989 & 870.61 & 3 & $30, \mathrm{II}$ & $a^{5} \overline{\mathrm{F}}_{2}-a^{5} \overline{\mathrm{G}}_{2}$ & & $\begin{array}{r}00.201 \\
3899.711\end{array}$ & 635.69 & 10 & $30 \mathrm{R}, \mathrm{I}$ & $a^{5} \mathrm{D}_{2}-a^{5} \overline{\mathrm{D}}_{2}$ & \\
\hline 3608.860 & 701.70 & 30 & $100 \mathrm{r}, \mathrm{I}$ & $a^{5} \overline{\bar{F}}_{1}-a^{5} \overline{\mathrm{G}}_{2}$ & & 3899.711 & $\begin{array}{l}030.09 \\
501.31\end{array}$ & 10 & $\begin{array}{r}30 \mathrm{~K}, 1 \\
20 \mathrm{r}, \mathrm{r}\end{array}$ & $a^{5} \mathrm{D}_{0}-a^{5} \overline{\mathrm{D}}_{1}$ & \\
\hline 18. 769 & 625.84 & 40 & $125 \mathrm{R}, \mathrm{I}$ & $a^{5} \overline{\mathrm{F}}_{2}-a^{5} \overline{\mathrm{G}}_{3}$ & & $\begin{array}{r}3920.201 \\
22.916\end{array}$ & $\begin{array}{l}501.31 \\
484.03\end{array}$ & 1 & $\begin{array}{r}20 \mathrm{r}, \mathrm{I} \\
25 \mathrm{R}, \mathrm{I}\end{array}$ & $\begin{array}{l}a^{0} \mathrm{D}_{0}-a^{5} \mathrm{D}_{1} \\
a^{5} \mathrm{D}_{3}-a^{5} \overline{\mathrm{D}}_{4}\end{array}$ & \\
\hline 31.464 & 529.29 & 50 & $125 \mathrm{R}, \mathrm{I}$ & $a^{5} \overline{\mathrm{F}}_{3}-a^{5} \overline{\mathrm{G}}_{4}$ & & 27.925 & $\begin{array}{l}484.05 \\
451.55\end{array}$ & $\begin{array}{l}2 \\
2\end{array}$ & $\begin{array}{ll}25 & \mathrm{R}, \mathrm{1} \\
30 & \mathrm{R}, \mathrm{I}\end{array}$ & $\begin{array}{l}a^{0} \mathrm{D}_{3}-a^{5} \mathrm{D}_{4} \\
a^{5} \mathrm{D}_{1}-a^{5} \overline{\mathrm{D}}_{2}\end{array}$ & \\
\hline 47.845 & 405.65 & 40 & $100 \mathrm{R}, \mathrm{I}$ & $a^{5} \overline{\mathrm{F}}_{4}-a^{5} \overline{\mathrm{G}}_{5}$ & & & & & & & \\
\hline 79.915 & 166.84 & 5 & $40 \mathrm{r}, \mathrm{I}, \mathrm{A}$ & $a^{5} \mathrm{D}_{4}-a^{5} \mathrm{~F}_{4}$ & & 30.304 & 25436.15 & 2 & $25 \mathrm{R}, \mathrm{I}$ & $a^{5} \underline{\mathrm{D}}_{2}-a^{5} \overline{\mathrm{D}_{3}} 3$ & \\
\hline 3687.458 & 27111.27 & 3 & $40 \mathrm{r}, \mathrm{I}$ & $a^{5} \overline{\mathrm{F}}_{5}-b^{5} \overline{\mathrm{F}}_{4}$ & & 45.822 & 24709.90 & $20 p$ & $60 \mathrm{r}, \mathrm{II}$ & $a^{3} \bar{F}_{4}-a^{3} \bar{F}_{4}$ & \\
\hline 3705.567 & 26978.77 & 10 & $100 \mathrm{r}, \mathrm{I}$ & $a^{5} \mathrm{D}_{3}-a^{5} \mathrm{~F}_{3}$ & & 63.604 & 601.78 & $10 p$ & $45, \mathrm{II}$ & $a^{3} \overline{\mathrm{F}}_{3}-a^{5} \mathrm{~F}_{3}$ & \\
\hline 09.250 & 951.99 & 5 & $75 \mathrm{r}, \mathrm{II}$ & $a^{5} \overline{\mathbf{F}}_{4}-b^{5} \bar{F}_{3}$ & & 4071.748 & 24552.57 & $5 p$ & 40, II & $a^{3} \overline{\mathrm{F}}_{2}-a^{3} \mathrm{~F}_{2}$ & \\
\hline & & & & & & 4271.764 & 23402.96 & $3 p$ & $35, \mathrm{II}$ & $a^{3} \overline{\mathrm{F}}_{4}-a^{3} \overline{\mathrm{G}}_{5}$ & \\
\hline 19.938 & 874.55 & 100 & $250 \mathrm{R}, \mathrm{I}$ & $a^{5} \mathrm{D}_{4}-a^{5} \mathrm{~F}_{5}$ & $A, G$ & & & & & & \\
\hline 22.565 & 855.59 & 8 & $50 \mathrm{r}, \mathrm{I}, \mathrm{A}$ & $a^{5} \mathrm{D}_{2}-a^{5} \mathrm{~F}_{2}$ & & 4307.910 & 206.61 & $3 p$ & $35, \mathrm{II}$ & $a^{3} \overline{\mathrm{F}}_{3}-a^{3} \overline{\mathrm{G}}_{4}$ & \\
\hline 27.622 & 819.15 & 3 & $50 \mathrm{r}, \mathrm{II}$ & $a^{5} \overline{\mathrm{F}}_{3}-b^{5} \mathrm{~F}_{2}$ & & 25.770 & 23110.80 & $3 p$ & $35, \mathrm{II}$ & $a^{3} \overline{\mathrm{F}}_{2}-a^{3} \overline{\mathrm{G}}_{3}$ & \\
\hline 33.319 & 778.23 & 10 & $40 \mathrm{r}, \mathrm{I}, \mathrm{A}$ & $a^{5} \mathrm{D}_{1}-a^{5} \mathrm{~F}_{1}$ & & 4383.548 & 22806.18 & $10 p$ & $45 \mathrm{r}, \mathrm{II}$ & $a^{3} \overline{\mathrm{F}}_{4}-a^{5} \overline{\mathrm{G}}_{4}$ & \\
\hline 3734.869 & 26767.12 & 50 & $300 \mathrm{r}, \mathrm{II}$ & $a^{5} \overline{\mathrm{F}}_{5}-b^{5} \mathrm{~F}_{8}$ & & 4404. 752 & 22696.40 & $5 p$ & $30, \mathrm{II}$ & $a^{3} \overline{\mathrm{F}}_{3}-a^{5} \overline{\mathrm{G}}_{4}$ & \\
\hline
\end{tabular}


TABLE 2.-Low levels in spectra of iron

\begin{tabular}{|c|c|c|c|c|c|c|c|c|c|}
\hline $\begin{array}{l}\text { Spec- } \\
\text { trum }\end{array}$ & Value & $\begin{array}{l}\text { Differ- } \\
\text { ence }\end{array}$ & Symbol & Electrons & $\begin{array}{l}\text { Spec- } \\
\text { trum }\end{array}$ & Value & $\begin{array}{l}\text { Differ- } \\
\text { ence }\end{array}$ & Symbol & Electrons \\
\hline $\mathrm{Fe} \mathrm{I}$ & $\begin{array}{r}0.000 \\
415.936 \\
704.008 \\
888.140 \\
978.086 \\
6928.285 \\
7376.780 \\
7728.078 \\
7985.802 \\
8154.736 \\
11976.270 \\
12560.966 \\
12958.588 \\
17550.222 \\
17727.026 \\
17927.419\end{array}$ & $\begin{array}{r}415.936 \\
288.072 \\
184.132 \\
89.946 \\
448.495 \\
351.298 \\
257.724 \\
168.934\end{array}$ & $\begin{array}{l}a^{5} \mathrm{D}_{4} \\
a^{5} \mathrm{D}_{3} \\
a^{5} \mathrm{D}_{2} \\
a^{5} \mathrm{D}_{1} \\
a^{5} \mathrm{D}_{0} \\
a^{6} \overline{\mathrm{F}}_{5} \\
a^{5} \overline{\mathrm{F}}_{4} \\
a^{5} \overline{\mathrm{F}}_{3} \\
a^{5} \overline{\mathrm{F}}_{2} \\
a^{5} \overline{\mathrm{F}}_{1} \\
a^{3} \overline{\mathrm{F}}_{4} \\
a^{3} \overline{\mathrm{F}}_{3} \\
a^{3} \overline{\mathrm{F}}_{2} \\
a^{6} \overline{\mathrm{P}}_{2} \\
a^{5} \overline{\mathrm{P}}_{1} \\
a^{5} \overline{\mathrm{P}}_{0}\end{array}$ & $4 s, 3 d^{7}$ & Fe II & $\begin{array}{r}0.00 \\
384.80 \\
667.65 \\
862.55 \\
\cdot 976.96 \\
1872.56 \\
2430.16 \\
2837.91 \\
3117.49 \\
7955.24 \\
8391.90 \\
8680.37 \\
8846.72 \\
13474.36 \\
13673.04 \\
13904.74\end{array}$ & $\begin{array}{l}384.80 \\
282.85 \\
194.90 \\
114.41 \\
\\
557.60 \\
407.75 \\
279.58\end{array}$ & $\begin{array}{l}a^{6} \mathrm{D}_{5} \\
a^{6} \mathrm{D}_{4} \\
a^{6} \mathrm{D}_{3} \\
a^{6} \mathrm{D}_{2} \\
a^{6} \mathrm{D}_{1} \\
a^{4} \overline{\mathrm{F}}_{5} \\
a^{4} \overline{\mathrm{F}}_{4} \\
a^{4} \overline{\mathrm{F}}_{3} \\
a^{4} \overline{\mathrm{F}}_{2} \\
a^{4} \mathrm{D}_{4} \\
a^{4} \mathrm{D}_{3} \\
a^{4} \mathrm{D}_{2} \\
a^{4} \mathrm{D}_{1} \\
a^{4} \overline{\mathrm{P}}_{3} \\
a^{4} \overline{\mathrm{P}}_{2} \\
a^{4} \overline{\mathrm{P}}_{1}\end{array}$ & $4 s, 3 d^{6}$ \\
\hline
\end{tabular}

2. COBALT $(\mathrm{Co}=58.97 ; Z=27)$

Rods of cobalt metal about $3 \mathrm{~mm}$ square served as electrodes for underwater spark spectra. This metal contained a trace of nickel and a trace of copper; the well-known copper arc lines (3247 and $3274 \mathrm{~A}$ ) were regularly found absorbed in our spectrograms. Besides these, about 340 absorption lines were identified as belonging to cobalt, and 20 or more of them almost certainly originate with ionized atoms. The complete list of absorbed lines is given in Table 3, in which the wave lengths below $2300 \mathrm{~A}$ are quoted from Piña (22), between 2300 and 2640 A from Exner and Haschek (23), and the longer ones from Dhein (24). The relative intensities in arc spectra are quoted from the same observers except above $2990 \mathrm{~A}$, where King's (25) intensities and temperature classes begin. Lines certainly to be regarded as originating with ionized atoms are marked $\mathrm{Co}^{+}$; those observed by Angerer and Joos in furnace vapor absorption are noted with the letter A, while those obtained by Sur and Majumdar (26) from an absorption furnace are marked S. 
The structure of the Co I spectrum has been investigated by Walters (27) and by Catalán and Bechert (28). The combinations listed in Table 1 are in part those of Catalán and Bechert, but their notation has been revised. In addition, a considerable number of new levels have been found and confirmed by the absorption lines and other combinations. These new combinations are marked with asterisks.

The arc spectrum of cobalt is characterized by two quartet $F$ terms of low energy value, one of which comes from the electron configuration $\left(s^{2}, d^{7}\right)$ and represents the normal state of the atom. The other ${ }^{4} \mathrm{~F}$ lies about half a volt higher in the energy diagram; it, as well as the next higher ${ }^{2} \mathrm{~F}$ and ${ }^{4} \mathrm{P}$ terms, probably originates with the $\left(s, d^{8}\right)$ configuration. Both ${ }^{4} \mathrm{~F}$ terms are prominent in our absorption lines, but the ${ }^{2} \mathrm{~F}$ term is represented by weaker lines, most of which are only partially reversed.

More than 2,500 lines have been observed in the emission spectrum of the cobalt arc. About 14 per cent of these are seen absorbed in the underwater spark, and they are distributed as follows: 118 lines of total intensity 1,920 from $a^{4} \mathrm{~F}, 84$ lines of total intensity 1,490 from $b^{4} \mathrm{~F}$, and 21 lines with intensity sum 330 from $a^{2} \mathrm{~F}$. Thus, notwithstanding the relative closeness of metastable states to the normal one, the latter still has some advantage in the production of absorption lines, although the preference is not so marked as for Fe.

The structure of the Co II spectrum is being investigated by Meggers and Burns, and the combinations for $\mathrm{Co}^{+}$lines in Table 3 are quoted from their preliminary results. A low metastable state of ionized cobalt is represented by a quintet $F$ term arising from the configuration $\left(s, d^{7}\right)$ after one of the $s$ electrons of the normal atom has been removed. Practically all of the absorbed spark lines involve this term, and the additional fact that most of these lines have been observed self-reversed in the ordinary emission spectrum of a condensed spark between cobalt electrodes is experimental proof that $a^{5} \mathrm{~F}$ is a term with low energy. The normal state of $\mathrm{Co}^{+}$ is probably represented by a triplet $\mathrm{F}$ term originating with eight $d$ electrons (see Laporte, Zeitschr. f. Phys., 39, p. 123; 1926); it has not been identified because its chief combinations are expected to give shorter wave lengths than those in our list. 
TABLE 3.-Underwater spark absorption spectrum of cobalt

\begin{tabular}{|c|c|c|c|c|c|c|c|c|c|c|c|}
\hline \multirow{2}{*}{$\lambda$} & \multirow{2}{*}{$\nu$} & \multicolumn{2}{|c|}{$\begin{array}{l}\text { Intensities } \\
\text { and class }\end{array}$} & \multirow{2}{*}{$\begin{array}{c}\text { Combina- } \\
\text { tion }\end{array}$} & \multirow{2}{*}{ Notes } & \multirow{2}{*}{$\lambda$} & \multirow{2}{*}{$\nu$} & \multicolumn{2}{|c|}{$\begin{array}{l}\text { Intensities } \\
\text { and class }\end{array}$} & \multirow{2}{*}{$\underset{\text { tion }}{\text { Combina- }}$} & \multirow{2}{*}{ Notes } \\
\hline & & A & $\mathrm{E}$ & & & & & A & $\mathrm{E}$ & & \\
\hline 2137.82 & 46761.8 & 3 & 1 & $a^{4} \mathrm{~F}_{2}-32^{*}$ & \multirow{10}{*}{$\mathrm{Ni} ?$} & 2276.56 & 43912.3 & 15 & 6 & & A \\
\hline 46. 30 & 577.1 & 5 & 2 & $a^{4} \mathrm{~F}_{4}-18_{3} *$ & & 79.53 & 855.1 & 4 & 4 & $4 b^{4} \mathrm{~F}_{3}-20_{2}{ }^{*}$ & \multirow[b]{4}{*}{$\mathrm{A}, \mathrm{Co}^{+}$} \\
\hline 58.34 & 317.3 & 2 & 3 & & & 84.96 & 750.9 & 3 & 3 & $\begin{array}{l}34 F_{2}-19_{3} *\end{array}$ & \\
\hline 63.60 & 204.7 & 10 & 3 & $a^{4} \mathrm{~F}_{3}-31^{*}$ & & 85.50 & 740.6 & 3 & 3 & & \\
\hline 65.14 & 171.8 & 3 & 2 & $a^{4} \mathrm{~F}_{2}-30^{*}$ & & 86.21 & 727.0 & 10 & 5 & $5 \mid a^{5} \mathrm{~F}_{5}-a^{5} \mathrm{G}_{6} *$ & \\
\hline 66.86 & 135.2 & 5 & 2 & & & 87.87 & 695.3 & 20 & 6 & 6 $\quad a^{4} \mathrm{~F}_{4}-\mathrm{m}$ & A \\
\hline 68.79 & 46094.1 & 8 & 3 & $a^{4} \mathrm{~F}_{4}-29^{*}$ & & 90.1 & 652.7 & 4 & $?$ & ? $a^{4} \mathrm{~F}_{4}-24^{*}$ & $\mathrm{Ni} ?$ \\
\hline 73.89 & 45986.0 & 5 & 3 & $a^{4} \mathrm{~F}_{3}-18_{3}{ }^{*}$ & & 90.60 & 643.2 & 3 & 3 & & \\
\hline 74.44 & 974.4 & & 3 & & & 91.50 & 626.1 & 10 & 5 & & \\
\hline 74.67 & 969.5 & 50 & 3 & $a^{4} \mathrm{~F}_{5}-11_{4}^{*}$ & & 94.04 & 577.8 & 5 & 3 & $3 a^{4} \mathrm{~F}_{4}-25^{*}$ & \\
\hline & & & & & & 95.23 & 555.2 & 20 & 4 & $4 a^{4} \mathrm{~F}_{5}-84^{*}$ & \\
\hline 78.08 & 897.6 & 4 & 1 & & & 96.09 & 538.8 & 8 & 3 & & \\
\hline 78.92 & 879.9 & 5 & 1 & & & 96.74 & 526.5 & 10 & 3 & & \\
\hline 80.01 & 856.9 & 10 & 3 & $a^{4} \mathrm{~F}_{4}-15_{3}^{*}$ & & 2298.39 & 495.3 & 6 & 4 & $4 b^{4} \mathrm{~F}_{2}-32 *$ & \\
\hline 81.12 & 833.6 & 3 & 3 & & & 2300.82 & 449.4 & 2 & 1 & & \\
\hline 81.77 & 819.9 & 3 & 2 & & & 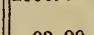 & & & & & \\
\hline & & & & & & 03.99 & 389.6 & 15 & 1 & $\begin{array}{l}b^{4} F_{3}-17_{4}^{*} \\
\left\{b^{4} F_{2}-19_{3}^{*}\right.\end{array}$ & \\
\hline $\begin{array}{l}82.57 \\
84.31\end{array}$ & $\begin{array}{l}803.2 \\
766.7\end{array}$ & $\begin{array}{l}2 \\
1\end{array}$ & $\begin{array}{l}3 \\
1\end{array}$ & $a^{4} \mathrm{~F}_{2}-31^{*}$ & & 05.18 & 367.2 & 10 & 1 & 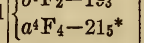 & \\
\hline 86.79 & 714.8 & 10 & 4 & & & 07.89 & 316.3 & 6 & & $a^{5} \mathrm{~F}_{4}-a^{5} \mathrm{G}_{5} *$ & $\mathrm{Co}^{+}$ \\
\hline 90.3 & 614.5 & 2 & 3. & & $\mathrm{Ni} ?$ & 09.03 & 294.9 & 30 & 1 & $a^{4} \mathrm{~F}_{5}-\mathrm{b}_{5}$ & \\
\hline 91.90 & 608.2 & 5 & 2 & & & 11.38 & 250.9 & & 1 & $1 b^{4} F_{4}-18_{3}^{*}$ & \\
\hline 96.59 & 510.8 & 40 & 5 & $a^{4} \mathrm{~F}_{4}-28^{*}$ & & 11.65 & 245.8 & 100 & 2 & $\left\{a^{4} \mathrm{~F}_{2}-1_{3}\right.$ & A \\
\hline 2199.66 & 447.3 & 5 & 4 & & & & & & & $\left(a^{5} \mathrm{~F}_{3}-a^{5} \mathrm{G}_{4}{ }^{*}\right.$ & \\
\hline 2204.9 & 339.3 & 5 & 3 & & & 13.67 & 208.1 & 6 & 1 & & \\
\hline 07.89 & 277.9 & 10 & 5 & $a^{4} \mathrm{~F}_{3}-16_{2}^{*}$ & & 14.07 & 200.6 & 5 & 2 & $a^{5} \mathrm{~F}_{2}-a^{5} \mathrm{G}_{3}{ }^{*}$ & $\mathrm{Co}^{+}$ \\
\hline 08.59 & 263.6 & 5 & 3 & $a^{4} \mathrm{~F}_{3}-15^{*}$ & & $\begin{array}{l}14.99 \\
16.12\end{array}$ & $\begin{array}{l}183.4 \\
162.4\end{array}$ & $\begin{array}{l}5 \\
6\end{array}$ & $\begin{array}{l}2 \\
1\end{array}$ & $a^{5} \mathrm{~F}_{1}-a^{5} \mathrm{G}_{2} *$ & $\mathrm{Co}^{+}$ \\
\hline 12.38 & 186.1 & 4 & 4 & $a^{4} \mathrm{~F}_{4}-123^{*}$ & & 16.88 & 148.2 & 8 & 1 & $1 a^{4} \mathrm{~F}_{3}-23^{*}$ & \\
\hline 13. 92 & 154.6 & 20 & 6 & $\left\{\begin{array}{l}a^{4} \mathrm{~F}_{4}-11_{4}^{*} \\
a^{4} \mathrm{~F}_{3}-14_{2}^{*}\end{array}\right.$ & & 17.15 & 143.2 & 3 & 1 & & Ni? \\
\hline 19.27 & 45045.8 & 1 & 2 & & & 20.04 & 089.4 & 20 & 1 & & Ni? \\
\hline 25.43 & 44921.1 & & 3. & $a^{4} \mathrm{~F}_{3}-28^{*}$ & & 21.41 & 064.0 & 4 & 1 & $a^{4} \mathrm{~F}_{3}-24^{*}$ & \\
\hline 25.77 & 914.3 & $10 \mathrm{~d}$ & 3 & & & 23.18 & 031.2 & 25 & 1 & $a^{4} \mathrm{~F}_{4}-\mathrm{c}_{4}$ & A \\
\hline & & & & & & 24.36 & 43009.4 & 3 & 2 & $2 a^{5} \mathrm{~F}_{4}-a^{5} \overline{\mathrm{F}} 3^{*}$ & $\mathrm{Co}^{+}$ \\
\hline 27.84 & 872.5 & 8 & 4 & & & 25.55 & 42987.4 & 15 & 1 & $a^{4} \mathrm{~F}_{3}-25^{*}$ & \\
\hline 29.87 & 831.7 & 8 & 3 & $a^{4} \mathrm{~F}_{5}-27^{*}$ & & 26.15 & 976.3 & 2 & 2 & $a^{5} \mathrm{~F}_{3}-a^{5} \overline{\mathrm{F}}_{4} *$ & $\mathrm{Co}^{+}$ \\
\hline 32.54 & 778.1 & 2 & 3 & $a^{4} F_{3}-132^{*}$ & & 26.47 & 970.4 & 4 & 2 & $a^{5} \mathrm{~F}_{5}-a^{5} \overline{\mathrm{F}}_{4} *$ & $\mathrm{Co}^{+}$ \\
\hline 33.83 & 752.2 & 3 & 3 & $a^{4} \mathrm{~F}_{2}-14_{2}{ }^{*}$ & & 29.12 & 921.5 & 4 & 1 & $b^{4} \mathrm{~F}_{3}-31^{*}$ & \\
\hline 34.86 & 731.6 & 4 & 4 & & & & & & & & \\
\hline & & & & & & 29.97 & 905.8 & 4 & 1 & $b^{4} \mathrm{~F}_{2}-30^{*}$ & \\
\hline 36.84 & 692.0 & 15 & 4 & & & 32.2 & 864.8 & 2 & ? & & \\
\hline 45.17 & 526.2 & 1 & 4 & & & 33.1 & 848.3 & 2 & $?$ & $b^{4} F_{5}-28^{*}$ & \\
\hline 46.61 & 497.7 & 2 & 3 & $a^{4} \mathrm{~F}_{3}-10_{2}{ }^{*}$ & & 34.2 & 838.1 & 1 & ? & & \\
\hline 52.77 & 376.0 & 3 & 3 & $a^{4} \mathrm{~F}_{2}-13_{2} *$ & & 35.98 & 795.4 & 20 & 1 & $a^{4} \mathrm{~F}_{3}-\mathrm{i}_{3}$ & A \\
\hline 53.6 & 359.7 & & 3 & & Ni? & 37.49 & 767.8 & 1 & 1 & $b^{4} \mathbf{F}_{4}-29^{*}$ & \\
\hline 53.91 & 353.6 & 4 & 3 & & & 38.0 & 758.5 & 7 & $?$ & & \\
\hline 56.64 & 299.9 & 5 & 4 & $b^{4} \mathrm{~F}_{4}-193^{*}$ & & 38.65 & 746.6 & 8 & 1 & $a^{4} \mathrm{~F}_{2}-23^{*}$ & \\
\hline 57.64 & 280.3 & 2 & 3 & $a^{4} \mathrm{~F}_{4}-26^{*}$ & & 39.05 & 739.3 & 6 & 1 & $a^{4} F_{4}-8_{4}^{*}$ & \\
\hline 62.63 & 182.6 & 2 & 3 & $a^{4} \mathrm{~F}_{5}-21_{5}^{*}$ & & 44. 29 & 643.8 & 2 & 1 & $\left\{a^{4} \mathrm{~F}_{2}-\mathrm{n}_{1}\right.$ & a \\
\hline 64. 96 & 137.2 & 3 & 3 & & & & & & & {$\left[a^{5} \mathrm{~F}_{1}-a^{5} \mathrm{~F}_{1}{ }^{*}\right]$} & $\mathrm{Co}^{+}$ \\
\hline & & & & & & 45.57 & 620.5 & 8 & 1 & & \\
\hline 67.17 & 094.2 & 1 & 2 & $a^{4} \mathrm{~F}_{2}-102^{*}$ & & 46.18 & 609.4 & 10 & 1 & $a^{4} \mathrm{~F}_{5}-a_{5}$ & A \\
\hline 68.24 & 073.3 & 6 & 3 & & & & 586 & & & $\left\{{ }^{4} a F_{2}-25^{*}\right.$ & \\
\hline 68.83 & 44061.9 & 1 & 3 & & & 47.40 & 000.7 & $0, \mathrm{a}$ & & {$\left[a^{5} \mathrm{~F}_{2}-a^{6} \overline{\mathrm{F}_{2}} 2^{*}\right]$} & $\mathrm{Co}^{+}$ \\
\hline 74. 60 & 43950.2 & 8 & 5 & & $\mathbf{A}$ & 50.32 & 534.4 & 6 & 1 & $b^{4} \mathrm{~F}_{2}-31^{*}$ & \\
\hline 2275.92 & 43924.7 & 4 & $3 \mid$ & & A & $\|_{2351.41}$ & $\mid 42514.6$ & $6 \mid$ & 1 & 1) $a^{4} F_{3}-93^{*}$ & \\
\hline
\end{tabular}


TABLE 3.-Underwater spark absorption spectrum of cobalt-Continued

\begin{tabular}{|c|c|c|c|c|c|c|c|c|c|c|c|}
\hline \multirow{2}{*}{$\lambda$} & \multirow{2}{*}{$\nu$} & \multicolumn{2}{|c|}{$\begin{array}{l}\text { Intensities } \\
\text { and class }\end{array}$} & \multirow{2}{*}{$\begin{array}{c}\text { Combina- } \\
\text { tion }\end{array}$} & \multirow{2}{*}{ Notes } & \multirow{2}{*}{$\lambda$} & \multirow{2}{*}{$\nu$} & \multicolumn{2}{|c|}{$\begin{array}{l}\text { Intensities } \\
\text { and class }\end{array}$} & \multirow{2}{*}{$\underset{\text { tion }}{\text { Combina- }}$} & \multirow{2}{*}{ Notes } \\
\hline & & A & $\mathrm{E}$ & & & & & A & $\mathbf{E}$ & & \\
\hline 2352.89 & 42487.9 & 10 & 1 & $b^{4} \mathrm{~F}_{6}-11_{4}^{*}$ & & 2463. 77 & 40575.9 & 2 & 1 & & \\
\hline 53.43 & 478.2 & 10 & 2 & $\left\{\begin{array}{l}a^{4} \mathrm{~F}_{4}-b_{5} \\
a^{6} \mathrm{~F}_{3}-a^{5} \overline{\mathrm{F}}_{3}\end{array}\right.$ & $\begin{array}{l}\mathrm{A} \\
\mathrm{Co}^{+}\end{array}$ & $\begin{array}{l}64.50 \\
64.63\end{array}$ & \begin{tabular}{|l|}
563.9 \\
561.8
\end{tabular} & 3 & $\begin{array}{l}1 \\
1\end{array}$ & $a^{4} \mathrm{~F}_{3}-22^{*}$ & \\
\hline 54.50 & 458.8 & 8 & ? & $a^{4} \mathrm{~F}_{3}-\mathrm{c}_{4}$ & A & 67.72 & 511.0 & 6 & 1 & $a^{4} \mathrm{~F}_{3}-c^{4} \bar{F}_{4}$ & \\
\hline 58.21 & 392.1 & 7 & 1 & $a^{4} \mathrm{~F}_{2}-\mathrm{i}_{3}$ & & 70.30 & 468.7 & 6 & 1) & & \\
\hline 63.81 & 291.6 & 6 & 2 & $a^{5} \mathrm{~F}_{4}-a^{5} \overline{\mathrm{F}}^{4}$ & $\mathrm{Co}^{+}$ & 72.94 & 425.5 & 1 & 1 & & - \\
\hline 65.04 & 269.6 & 20 & 1 & $a^{4} \mathrm{~F}_{6}-c^{4} \mathrm{G}_{5}$ & A & 73.93 & 409.3 & 1 & 1 & $a^{4} \mathrm{~F}_{4}-c^{4} \overline{\mathrm{F}}_{8}$ & \\
\hline 69.68 & 186.9 & 10 & 1 & $b^{4} \mathrm{~F}_{4}-28^{*}$ & & 76.64 & 365.1 & 10 & 1 & $\left\{b^{4} \mathrm{~F}_{6}-\mathrm{c}_{4}\right.$ & \\
\hline 70.52 & 171.9 & 6 & 1 & & & 0.02 & 000.4 & - & 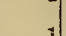 & $\left(b^{4} \mathrm{~F}_{3}-l_{3}\right.$ & \\
\hline 71.86 & 148.1 & 5 & 1 & $a^{4} \mathrm{~F}_{3}-84^{*}$ & & 83.61 & 251.8 & 20 & 1 & $b^{4} \mathrm{~F}_{4}-25^{*}$ & \\
\hline 72.87 & 130.2 & 3 & 1 & $a^{4} \mathrm{~F}_{3}-73^{*}$ & & 88.46 & 173.4 & 8 & 1 & $a^{4} \mathrm{~F}_{2}-4_{2}{ }^{*}$ & \\
\hline 77.22 & 053.1 & 4 & 1 & & & 89.27 & 160.3 & 2 & 1 & $a^{4} \mathrm{~F}_{2}-3_{1}^{*}$ & \\
\hline 78.62 & 42028.3 & 8 & 2 & $a^{5} \mathrm{~F}_{6}-a^{5} \mathrm{D}_{4}^{*}$ & $\mathrm{Co}^{+}$ & 93. 96 & 084.8 & 2 & 1 & $a^{2} \mathrm{~F}_{3}-2 \mathrm{2}^{*}$ & \\
\hline 80.52 & 41994.8 & 15 & 1 & $\left\{\begin{array}{l}a^{4} \mathrm{~F}_{4}-c^{4} \mathrm{G}_{4} \\
b^{4} \mathrm{~F}_{3}-16_{2}^{*}\end{array}\right.$ & A & $\begin{array}{l}94.74 \\
95.56\end{array}$ & $\begin{array}{l}072.2 \\
059.1\end{array}$ & $\begin{array}{l}2 \\
4\end{array}$ & $\begin{array}{l}1 \\
1\end{array}$ & $\begin{array}{l}b^{4} \mathbf{F}_{6}-8_{4}^{*} \\
b^{4} \mathbf{F}_{4}-i_{3}\end{array}$ & \\
\hline 83.47 & 942.8 & 5 & 2 & $a^{6} \mathrm{~F}_{4}-a^{5} \mathrm{D}_{3} *$ & $\mathrm{Co}^{+}$ & 2496.71 & 40040.6 & 5 & 1 & $a^{4} \mathrm{~F}_{4}-21_{5}^{*}$ & \\
\hline 84.89 & 917.9 & 20 & 1 & $a^{4} \bar{F}_{6}-c^{4} \overline{F_{4}}$ & A & 2504.54 & 39915.5 & 8 & 1 & $a^{4} \mathrm{~F}_{2}-64^{*}$ & \\
\hline 86.38 & 891.7 & 5 & 2 & $a^{5} \mathrm{~F}_{3}-a^{5} \mathrm{D}_{2} *$ & $\mathrm{Co}^{+}$ & 06.46 & 884.9 & 5 & 2 & & \\
\hline 87.47 & 872.6 & 4 & 1 & $b^{4} \mathrm{~F}_{3}-14_{2}{ }^{*}$ & & 07. 17 & 873.6 & & 1 & & \\
\hline 88.17 & 860.3 & & 1 & $b^{4} \mathrm{~F}_{4}-12^{*}$ & - & 07.7 & 865.2 & 4 & $?$ & & \\
\hline 88.40 & 856.3 & $\mathrm{sa}$ & 1 & $a^{4} \mathrm{~F}_{3}-22^{*}$ & 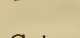 & 11.03 & 812.3 & 20 & 2 & $b^{4} \mathrm{~F}_{5}-\mathrm{b}_{5}$ & \\
\hline 88.93 & 847.0 & 8 & 2 & $a^{5} \mathrm{~F}_{6}-a^{5} \overline{\mathrm{F}}^{*}$ & $\mathrm{Co}^{+}$ & 12. 92 & 782.4 & 5 & 1 & $b^{4} \mathrm{~F}_{3}-24^{*}$ & \\
\hline 89.55 & 836.1 & 3 & 2 & $\left\{\begin{array}{l}a^{4} \mathrm{~F}_{3}-5_{3}^{*} \\
a^{5} \mathrm{~F}_{2}-a^{5} \mathrm{D}_{1}^{*}\end{array}\right\}$ & $\mathrm{Co}^{+}$ & 13. 11 & $\begin{array}{l}779.4 \\
705.1\end{array}$ & 8 & $\begin{array}{l}1 \\
2\end{array}$ & $\begin{array}{l}b^{4} \mathrm{~F}_{4}-9_{3}^{*} \\
b^{4} \mathrm{~F}_{4}-c_{4}\end{array}$ & \\
\hline $\begin{array}{l}90.01 \\
91.43\end{array}$ & 828.1 & 3 & 1 & $b^{4} \mathrm{~F}_{4}-11^{*}$ & & $\begin{array}{l}21.40 \\
28.97\end{array}$ & 648.6 & 100 & $\begin{array}{lll}3 & \mathrm{u}\end{array}$ & $a^{4} \mathrm{~F}_{5}-c^{4} \mathrm{D}_{4}$ & A \\
\hline 91.43 & 803.2 & 5 & 1 & $\int a^{4} F_{4}-a_{5}$ & & 28.97 & 529.9 & 70 & $3 \mathrm{u}$ & $a^{4} \mathrm{~F}_{4}-c^{4} \mathrm{D}_{3}$ & $A, B$ \\
\hline 2397. 37 & 793.4 & 10 & 1 & $\left\{a^{4} \mathrm{~F}_{3}-c^{4} \mathrm{G}_{3}\right\}$ & A & $\begin{array}{l}30.13 \\
32.17\end{array}$ & 511.8 & 3 & 1 & $b^{4} \mathrm{~F}_{3}-\mathrm{i}_{3}$ & \\
\hline 2397.37 & 699.7 & 2 & 1 & & $\mathrm{Co}^{+}$ & $\begin{array}{l}32.17 \\
35.93 \\
44.25\end{array}$ & $\begin{array}{l}479.9 \\
421.4\end{array}$ & $\begin{array}{r}5 \\
50 \\
50\end{array}$ & $\begin{array}{l}2 \\
2 \\
2\end{array}$ & $\begin{array}{c}b^{4} \mathrm{~F}_{2}-23^{*} \\
a^{4} \mathrm{~F}_{3}-c^{4} \mathrm{D}_{2}\end{array}$ & A \\
\hline $\begin{array}{r}2402.12 \\
04.17\end{array}$ & 617.2 & 25 & 1 & $a^{4} \mathrm{~F}_{4}-c^{4} \overline{\mathrm{F}}_{3}$ & A & $\begin{array}{l}44.25 \\
44.85\end{array}$ & $\begin{array}{l}292.5 \\
283.3\end{array}$ & $\begin{array}{r}30 \\
2\end{array}$ & 2 & $a^{4} \mathrm{~F}_{2}-c^{4} \mathrm{D}_{1}$ & \\
\hline $\begin{array}{l}04.17 \\
07.27\end{array}$ & $\begin{array}{l}518.7 \\
528.2\end{array}$ & $\begin{array}{r}1 \\
150\end{array}$ & $\begin{array}{r}2 \\
3 \mathrm{u}\end{array}$ & & $\begin{array}{l}\mathrm{Co}^{+} \\
\mathrm{A}, \mathrm{S}\end{array}$ & & & & 1 & & \\
\hline 11.65 & $\begin{array}{l}528.2 \\
452.8\end{array}$ & 100 & $\begin{array}{ll}3 & u \\
3 & u\end{array}$ & $\begin{array}{l}a^{4} \mathrm{~F}_{5}-c^{4} \mathrm{G}_{8} \\
a^{4} \mathrm{~F}_{4}-c^{4} \mathrm{G}_{5}\end{array}$ & A & $\begin{array}{l}48.31 \\
49.28\end{array}$ & 229.9 & 5 & 2 & $a^{2} F_{4}-15_{3} *$ & \\
\hline 14.47 & 404.4 & & $3 \mathrm{u}$ & $a^{4} \mathrm{~F}_{3}-c^{4} \mathrm{G}_{4}$ & A & $\begin{array}{l}49.28 \\
53.00\end{array}$ & 215.0 & 3 & 1 & & \\
\hline 15. 32 & 389.8 & 100 & & $\left\{a^{4} \mathrm{~F}_{2}-c^{4} \mathrm{G}_{3}\right\}$ & & $\begin{array}{l}53.00 \\
53.35\end{array}$ & 157.9 & 5 & 2 & $b^{4} \mathbf{F}_{3}-c_{4}$ & \\
\hline & & & $3 \mathrm{u}$ & $\left\{\begin{array}{l}a^{4} \mathrm{~F}_{3}-c^{4} \overline{\mathrm{F}_{2}}\end{array}\right.$ & A & & 152.5 & 5 & 2 & $b^{4} \mathrm{~F}_{4}-\mathrm{b}_{5}$ & \\
\hline 17.67 & 349.6 & 4 & 2 & $\left\{b^{4} \mathrm{~F}_{5}-27^{*}\right.$ & & 55.06 & 126.3 & 4 & 2 & $\left\{\begin{array}{l}b^{4} \mathrm{~F}_{5}-\mathrm{ab}_{5} \\
b^{4} \mathrm{~F}_{2}-\mathrm{i}_{3}\end{array}\right.$ & \\
\hline 19.15 & 324.3 & 10 & 1 & $\left(a^{5} \mathrm{~F}_{4}-a^{5} \mathrm{D}_{4}^{*}\right.$ & $\mathrm{Co}^{+}$ & 56.76 & 100.3 & 3 & 2 & $b^{4} \mathrm{~F}_{4}-5_{3} *$ & \\
\hline 24.98 & 224.9 & 50 & $3 \mathrm{u}$ & $a^{4} \mathrm{~F}_{b}-c^{4} \overline{\mathrm{F}}_{5}$ & $A, S$ & $\begin{array}{l}59.39 \\
62.13\end{array}$ & 060.1 & 1 & 2 & & \\
\hline 32.20 & 102.6 & 40 & $3 u$ & $a^{4} \mathrm{~F}_{4}-c^{4} \overline{\mathrm{F}}_{4}$ & A & $\begin{array}{l}62.13 \\
64.05\end{array}$ & 39018.3 & 20 & 2 & $a^{4} \mathrm{~F}_{2}-c^{4} \mathrm{D}_{2}$ & \\
\hline 35.11 & & & & & & $\begin{array}{l}64.05 \\
67.33\end{array}$ & $\begin{array}{r}38989.1 \\
939.3\end{array}$ & $\begin{array}{r}2 \\
30\end{array}$ & $\begin{array}{l}2 \\
2\end{array}$ & $a^{4} \mathrm{~F}_{3}-\mathrm{c}^{4} \mathrm{D}_{3}$ & $\mathrm{Co}^{+}$ \\
\hline $\begin{array}{l}35.11 \\
36.43\end{array}$ & $\begin{array}{l}053.4 \\
031.2\end{array}$ & 1 & 3 & & & & 800.0 & 30 & & $a^{2} \mathrm{~F}_{3}-c^{2} \mathrm{D}_{3}$ & \\
\hline $\begin{array}{l}30.40 \\
36.58\end{array}$ & $\begin{array}{l}031.2 \\
028.7\end{array}$ & 30 & 1 & & & 72.23 & 865.1 & 2 & 1 & $b^{4} \mathrm{~F}_{3}-8_{4}^{*}$ & \\
\hline 36. 77 & 41025.5 & 30 & 1 & $b^{2} \mathrm{~F}_{6}-\mathrm{m}$ & & 73.471 & 846.40 & 7 & $2 ?$ & & \\
\hline 39.03 & 40987.5 & 20 & $\begin{array}{l}1 \\
1\end{array}$ & $a^{4} \mathrm{~F}_{3}-c^{4} \mathrm{~F}_{3}$ & A & 74. 36 & 833.0 & 25 & 2 & $a^{4} \mathrm{~F}_{4}-c^{4} \mathrm{D}_{4}$ & $\mathbf{S}$ \\
\hline & & & 1 & $a^{4} \mathrm{~F}_{2}-c^{4} \mathrm{~F}_{2}$ & A & 75. 73 & 812.3 & 1 & 1 & $a^{4} \mathrm{~F}_{2}-1_{2}{ }^{*}$ & \\
\hline 41.07 & 953.2 & 2 & 2 & $b^{4} \mathrm{~F}_{4}-26^{*}$ & & 80.34 & 743.0 & 4. & 3 & & $\mathrm{Co}^{+}$ \\
\hline 42.92 & 922.2 & 3 & 1 & & & 80.86 & 735.2 & 4 & 2 & & \\
\hline 43.55 & 911.7 & & 1 & $b^{4} \mathrm{~F}_{4}-\mathrm{l}_{3}$ & & 82.26 & 714.2 & 2 & 2 & & $\mathrm{Co}^{+}$ \\
\hline 56.23 & 700.5 & 5) & 1 & $b^{4} \mathrm{~F}_{5}-21 b^{*}$ & & 85.36 & 667.8 & 3 & 2 & $b^{4} F_{4}-c^{4} G_{4}$ & \\
\hline 2460.82 & 40624.6 & 5) & 1) & $\left.a^{4} \mathrm{~F}_{2}-c^{4} \overline{\mathrm{F}}_{3}\right]$ & & 2587.21 & $|38640.1|$ & 2 & 2 & & $\mathrm{Co}^{+}$ \\
\hline
\end{tabular}


TABLE 3.-Underwater spark absorption spectrum of cobalt-Continued

\begin{tabular}{|c|c|c|c|c|c|c|c|c|c|c|c|}
\hline \multirow{2}{*}{$\lambda$} & \multirow{2}{*}{$\nu$} & \multicolumn{2}{|c|}{$\begin{array}{l}\text { Intensities } \\
\text { and class }\end{array}$} & \multirow{2}{*}{$\underset{\text { tion }}{\text { Combina- }}$} & \multirow{2}{*}{ Notes } & \multirow{2}{*}{$\lambda$} & \multirow{2}{*}{$\nu$} & \multicolumn{2}{|c|}{$\begin{array}{l}\text { Intensities } \\
\text { and class }\end{array}$} & \multirow{2}{*}{$\begin{array}{c}\text { Combina- } \\
\text { tion }\end{array}$} & \multirow{2}{*}{ Notes } \\
\hline & & A & $\mathbf{E}$ & & & & & A & $\mathbf{E}$ & & \\
\hline 2590.61 & 38589.4 & 3 & 2 & & & 3086.778 & 32386.87 & 10 & $15 \mathrm{r}, \mathrm{II}$ & $\mid a^{4} \mathrm{~F}_{2}-b^{4} \overline{\mathrm{F}}_{2}$ & \\
\hline 91.69 & 573. 3 & 3 & 1 & $b^{4} F_{3}-22^{*}$ & & 89.593 & 357.36 & 6 & $10, \mathrm{II}$ & $a^{4} \mathrm{~F}_{4}-b^{4} \mathrm{G}_{4}$ & \\
\hline 2594.17 & 536.5 & 2 & 1 & $a^{4} \mathrm{~F}_{2}-c^{4} \mathrm{D}_{3}$ & & 3098.195 & 267.52 & 1 & $10, \mathrm{II}$ & $a^{4} \mathrm{~F}_{3}-b^{4} \mathrm{G}_{3}$ & \\
\hline 2600.98 & 435.6 & 1 & 1 & $b^{4} \mathrm{~F}_{3}-c^{4} \overline{\mathrm{F}}_{4}$ & & $\mid \begin{array}{r}3110.817 \\
18.240\end{array}$ & $\begin{array}{l}136.61 \\
060.10\end{array}$ & $\begin{array}{r}1 ? \\
1\end{array}$ & $\begin{array}{r}5, \mathrm{I} \\
5, \mathrm{II}\end{array}$ & $\begin{array}{l}a^{4} \mathrm{~F}_{2}-b^{4} \overline{\mathrm{F}}_{3} \\
a^{4} \mathrm{~F}_{3}-b^{4} \overline{\mathrm{F}}_{4}^{4}\end{array}$ & \\
\hline 06.13 & 359.6 & 4 & 1 & & & & & & & & \\
\hline 13.89 & 245.8 & 4 & 1 & & & 21.414 & 027. 51 & 18 & 10, II & $a^{4} F_{8}-b^{4} D_{4}$ & \\
\hline 14.14 & 242.1 & 2 & 1 & $a^{4} \mathrm{~F}_{3}-c^{4} \mathrm{D}_{4}$ & & 21.560 & 32026.01 & & $10, \mathrm{II}$ & $a^{4} \mathrm{~F}_{4}-b^{4} \mathrm{~F}_{5}$ & \\
\hline 17.86 & 187.8 & 2 & 1 & $b^{4} \mathrm{~F}_{2}-22^{*}$ & & $\begin{array}{l}37.325 \\
39.943\end{array}$ & $\begin{array}{r}31865.08 \\
838.52\end{array}$ & $\begin{array}{l}5 \\
5\end{array}$ & $\begin{array}{r}10, \mathrm{II} \\
12, \mathrm{II}\end{array}$ & $\begin{array}{l}a^{4} \mathrm{~F}_{2}-b^{4} \mathrm{G}_{8} \\
a^{4} \mathrm{~F}_{4}-b^{4} \mathrm{D}_{3}\end{array}$ & $S$ \\
\hline 19.27 & 167.2 & 2 & 1 & $b^{4} F_{2}-53^{*}$ & & 47.060 & 766.52 & 10 & $15, \mathrm{r}$ II & $a^{4} \mathrm{~F}_{3}-b^{4} \mathrm{C}_{4}$ & \\
\hline 22.06 & 126.6 & & 1 & $b^{4} \mathrm{~F}_{4}-c^{4} \mathrm{C}_{5}$ & & 49. 304 & 743.89 & 1 & $10, \mathrm{II}$ & $a^{4} \mathrm{~F}_{3}-b^{4} \mathrm{D}_{2}$ & \\
\hline 22.26 & 123.7 & 15 & 1 & $b^{4} \mathrm{~F}_{2}-c^{4} \mathrm{Cl}_{3}$ & & 54.785 & 688.74 & 5 & $10, \mathrm{II}$ & & \\
\hline 22.45 & 120.9 & & 1 & $b^{4} \mathrm{~F}_{3}-c^{4} \mathrm{G}_{4}$ & & 58. 769 & 648.77 & 5 & $12, \mathrm{II}$ & $a^{4} \mathrm{~F}_{4}-b^{4} \mathrm{G}_{5}$ & S \\
\hline 27.61 & 38046.1 & 10 & 2 & $b^{4} F_{3}-c^{4} G_{6}$ & & 3159.660 & 639.85 & 2 & $10, \mathrm{II}$ & $\left\{\begin{array}{l}a^{4} \mathrm{~F}_{2}-b^{4} \mathrm{D}_{1} \\
a^{4} \mathrm{~F}_{3}-b^{2} \mathrm{~F}_{3}\end{array}\right.$ & \}s \\
\hline 46.420 & 37775.66 & 4 & 4 & $b^{4} \mathrm{~F}_{4}-c^{4} \overline{\mathrm{F}}_{4}$ & & $|3219.155|$ & 31055.11 & $1 ?$ & $5, \mathrm{II}$ & $a^{4} \mathrm{~F}_{4}-a^{2} \mathrm{~F}_{4}$ & \\
\hline 48. 648 & 743.89 & 10 & 4 & $\left\{\begin{array}{l}b^{4} \bar{F}_{5}-c^{4} \bar{F}_{5} \\
b^{4} F_{3}-c^{4} \bar{F}_{3}\end{array}\right.$ & & 32.890 & 30923.18 & 1 & & & \\
\hline 49.940 & 725.49 & 1 & 3 & $a^{2} \mathrm{~F}_{3}-13_{2}{ }^{*}$ & & 37.028 & 883.65 & 3 & $8, \mathrm{II}$ & $a^{4} F_{4}-b^{2} G_{5}$ & \\
\hline 50.271 & 720.77 & 3 & 4 & $b^{4} \mathrm{~F}_{2}-c^{4} \overline{\mathrm{F}}_{2}$ & $\mathrm{~S}$ & $\begin{array}{r}43.840 \\
3283.452\end{array}$ & $\begin{array}{l}818.80 \\
447.02\end{array}$ & $\begin{array}{l}1 \\
4\end{array}$ & $\begin{array}{r}8, \text { II } \\
9, \text { I }\end{array}$ & & \\
\hline 75.987 & 358.30 & 2 & 4 & $b^{4} \mathrm{~F}_{2}-c^{4} \overline{\mathrm{F}}_{3}$ & & $\mid 3322.206$ & 30091.85 & 1 & $8, \mathrm{III}$ & 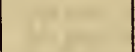 & \\
\hline 79. 758 & 305.74 & 3 & 2 & & & 33.390 & 29990.90 & 1 & $10, I$ & $b^{4} \mathrm{~F}_{4}-b^{2} \mathrm{G}_{4}$ & \\
\hline 85.340 & 228.20 & 4 & 3 & $b^{4} \mathrm{~F}_{8}-c^{4} \overline{\mathrm{F}}_{4}$ & & 34.151 & 984.05 & 20 & $30, r$ II & $b^{4} \mathrm{~F}_{5}-b^{4} \overline{\mathrm{F}}^{4}$ & $S$ \\
\hline 2695.853 & 37083.03 & 7 & 4 & $b^{4} \mathrm{~F}_{4}-c^{4} \overline{\mathrm{F}}_{8}$ & & 54. 386 & 803.17 & 20 & $10, \mathrm{II}$ & $b^{4} \mathrm{~F}_{4}-b^{4} \overline{\mathrm{F}}_{3}$ & $s$ \\
\hline 2715. 993 & 36808.06 & 4 & 3 & 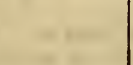 & & $\begin{array}{l}67.114 \\
70.330\end{array}$ & $\begin{array}{l}690.53 \\
662.20\end{array}$ & $\begin{array}{r}20 \\
2\end{array}$ & $\begin{array}{r}30 \mathrm{r}, \mathrm{II} \\
10, \mathrm{I}\end{array}$ & $\begin{array}{l}b^{4} \mathrm{~F}_{5}-b^{4} \mathrm{G}_{4} \\
b^{4} \mathrm{~F}_{3}-a^{2} \mathrm{D}_{2}\end{array}$ & S \\
\hline 31.116 & 604.2 & 1 & 4 & & & 85.227 & 531.67 & 10 & $25 \mathrm{r}, \mathrm{II}$ & $b^{4} \mathrm{~F}_{4}-b^{4} \mathrm{G}_{3}$ & $\mathrm{~S}$ \\
\hline 40.470 & 479. 32 & 2 & 3 & $a^{2} F_{4}-93^{*}$ & & 88.175 & 505. 98 & 15 & $30 \mathrm{r}, \mathrm{II}$ & $b^{4} \mathrm{~F}_{3}-b^{4} \overline{\mathrm{F}}_{2}$ & 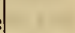 \\
\hline 45.108 & 417.69 & 2 & 4 & & & 3395.378 & 443.38 & 25 & $40 \mathrm{r}, \mathrm{II}$ & $b^{4} \mathrm{~F}_{3}-b^{2} \mathrm{G}_{4}$ & $\mathrm{~S}$ \\
\hline 50.10 & 351.6 & 1 & 1 & & & 3405.120 & 359.15 & 100 & $150 \mathrm{R}, \mathrm{II}$ & $b^{4} \mathrm{~F}_{5}-b^{4} \overline{\mathrm{F}_{5}}$ & A, S \\
\hline 61.375 & 203.17 & 2 & 3 & $b^{4} F_{4}-c^{4} D_{3}$ & & 09.176 & 324. 22 & 20 & $60 \mathrm{r}, \mathrm{II}$ & $b^{4} \mathrm{~F}_{4}-b^{4} \overline{F_{4}}$ & $\mathrm{~S}$ \\
\hline 64.193 & -166.26 & 4 & 4 & $b^{4} \mathrm{~F}_{6}-c^{4} \mathrm{D}_{4}$ & & 12. 335 & 297.07 & & $80 \mathrm{R}, \mathrm{II}$ & $b^{4} \mathrm{~F}_{4}-b^{2} \mathrm{G}_{5}$ & \\
\hline 66.225 & 36139.70 & 3 & 4 & $b^{4} F_{3}-c^{4} D_{2}$ & & 12.636 & 294.48 & $30 \mathrm{~d}$ & $80 \mathrm{R}, \mathrm{II}$ & $a^{4} \mathrm{~F}_{5}-a^{4} \mathrm{D}_{4}$ & $\int^{A, D}$ \\
\hline 78.830 & 35975.77 & 1 & 3 & & & 17.158 & 255. 72 & 30 & $50 \mathrm{r}, \mathrm{II}$ & $b^{4} \mathrm{~F}_{3}-b^{4} \overline{\mathrm{F}}_{3}$ & \\
\hline 2796. 236 & 751.84 & $1 ?$ & 3 & $b^{4} \mathrm{~F}_{2}-c^{4} \mathrm{D}_{2}$ & & 31.579 & 132. 79 & 30 & $50 \mathrm{r}, \mathrm{II}$ & $a^{4} F_{4}-a^{4} D_{3}$ & \\
\hline 2803.775 & 655.72 & 2 & 3 & $b^{4} \mathrm{~F}_{3}-c^{4} \mathrm{D}_{3}$ & & 33.043 & 120.36 & 40 & $60 \mathrm{R}, \mathrm{II}$ & $b^{4} \mathrm{~F}_{2}-b^{4} \overline{\mathrm{F}}_{2}$ & $\mathrm{~S}$ \\
\hline 15. 557 & 35506.52 & 2 & 4 & $b^{4} \mathrm{~F}_{4}-c^{4} \mathrm{D}_{4}$ & & 42. 924 & 036.79 & 30 & $40 \mathrm{r}, \mathrm{II}$ & $a^{4} \mathrm{~F}_{3}-a^{4} \mathrm{D}_{2}$ & \\
\hline 2886.448 & 34634.53 & 4 & 5 & $a^{4} \mathrm{~F}_{4}-b^{2} \overline{\mathrm{F}}_{4}$ & & 43.646 & 29030.70 & 50 & $80 \mathrm{R}, \mathrm{II}$ & $b^{4} \mathrm{~F}_{4}-b^{4} \mathrm{G}_{4}$ & A, S \\
\hline 2928.819 & 34133.49 & 1 & 3 & $a^{4} \mathrm{~F}_{3}-b^{2} \mathrm{G}_{4}$ & & 49. 171 & 28984.20 & $75 \mathrm{~d}$ & $60 \mathrm{R}, \mathrm{II}$ & $b^{4} \mathrm{~F}_{3}-b^{4} \mathrm{G}_{3}$ & \\
\hline 87.172 & 33466.75 & 10 & $15 \mathrm{r}, \mathrm{II}$ & $a^{4} \mathrm{~F}_{5}-b^{4} \overline{\mathrm{F}}_{4}$ & $\mathrm{~s}$ & 49.443 & 981.92 & & $60 \mathrm{R}, \mathrm{II}$ & $b^{4} \mathbf{F}_{5}-b^{4} \mathrm{G}_{5}$ & $\mathrm{~S}$ \\
\hline 2989.599 & 439.58 & 10 & $15 \mathrm{r}, \mathrm{II}$ & $a^{4} \mathrm{~F}_{5}-b^{2} \mathrm{G}_{b}$ & & 53.513 & 947.76 & 150 & $200 \mathrm{R}, \mathrm{II}$ & $b^{4} \mathrm{~F}_{5}-b^{4} \mathrm{G}_{8}$ & A, S \\
\hline 3000.554 & 317.50 & 1 & $7, \mathrm{II}$ & $a^{4} \mathrm{~F}_{4}-b^{2} \mathrm{G}_{4}$ & & $\begin{array}{l}55.236 \\
56.936\end{array}$ & $\begin{array}{l}933.32 \\
919.10\end{array}$ & $\begin{array}{r}10 \\
3\end{array}$ & \begin{tabular}{r|r}
$25 \mathrm{r}, \mathrm{I}$ \\
$9, \mathrm{I}$
\end{tabular} & $\begin{array}{l}a^{4} \mathrm{~F}_{2}-a^{4} \mathrm{D}_{1} \\
a^{4} \mathrm{~F}_{4}-a^{4} \mathrm{G}_{4}\end{array}$ & \\
\hline 13. 598 & $3 \quad 173.30$ & 3 & $8, \mathrm{II}$ & $a^{4} \mathrm{~F}_{3}-b^{4} \mathrm{G}_{4}$ & & 62.807 & 870.07 & 40 & $60 \mathrm{r}, \mathrm{II}$ & $b^{4} \mathrm{~F}_{2}-b^{4} \overline{\mathrm{F}}_{3}$ & $\mathrm{~S}$ \\
\hline 17. 552 & 33129.83 & 10 & $15 \mathrm{r}, \mathrm{II}$ & $a^{6} \mathrm{~F}_{4}-b^{4} \overline{\mathrm{F}}_{3}$ & & 65.796 & 845.17 & 50 & $100 \mathrm{R}, \mathrm{II}$ & $a^{4} \mathrm{~F}_{5}-a^{4} \mathrm{G}_{8}$ & $\mathrm{~A}, \mathrm{~S}$ \\
\hline 34.426 & 32945.60 & 1 & $6, \mathrm{II}$ & $a^{4} \mathrm{~F}_{3}-a_{2} \mathrm{D}_{2}$ & $S$ & & & & & $\left\{a^{4} \mathrm{~F}_{5}-a^{4} \overline{\mathrm{F}}_{4}\right.$ & \\
\hline 42.482 & 858.38 & 2 & $8, \mathrm{II}$ & $a^{4} \mathrm{~F}_{4}-b^{4} \mathrm{G}_{3}$ & & 74.019 & 776.89 & 60 & $100 \mathrm{R}, \mathrm{II}$ & $\left\{b^{4} \mathrm{~F}_{3}-b^{4} \frac{\mathrm{F}_{4}}{4}\right.$ & A, S \\
\hline 44.007 & 841.91 & 30 & $30 \mathrm{R}, \mathrm{II}$ & $a^{4} \mathrm{~F}_{5}-b^{4} \overline{\mathrm{F}_{5}}$ & $A, S$ & 83.415 & 699.28 & 15 & 5 $20, r, I$ & $b^{4} \mathrm{~F}_{4}-b^{4} \overline{\mathrm{F}}_{5}$ & $\mathrm{~S}$ \\
\hline 48. 892 & 789.29 & 5 & 12 r, II & $a^{4} \mathrm{~F}_{3}-b^{4} \overline{\mathrm{F}}_{2}$ & $\mathrm{~S}$ & 89,406 & 650.00 & 30 & $60 \mathrm{r}, \mathrm{II}$ & $a^{2} F_{4}-b^{2} D_{3}$ & $\mathrm{~S}$ \\
\hline 61.825 & 650.80 & 20 & $20 \mathrm{r}, \mathrm{II}$ & $a^{4} \mathrm{~F}_{4}-b^{4} \overline{\mathrm{F}}_{4}$ & $\mathrm{~S}$ & 90.741 & 639.04 & 2 & 2. $\quad 10, \mathrm{I}$ & $b^{4} \mathrm{~F}_{4}-a^{2} \overline{\mathrm{F}}_{3}$ & $\mathrm{~S}$ \\
\hline 72.346 & 539.00 & 15 & $15 \mathrm{r}, \mathrm{II}$ & $a^{4} \mathrm{~F}_{3}-b^{4} \overline{\mathrm{F}}_{3}$ & & 91.324 & 634.27 & 10 & $15, \mathrm{I}$ & $a^{4} \mathrm{~F}_{2}-a^{4} \mathrm{D}_{2}$ & $\mathrm{~S}$ \\
\hline 3082.614 & 32430.62 & 5 & $12 \mathrm{r}, \mathrm{II}$ & $a^{4} \mathrm{~F}_{5}-b^{4} \mathrm{G}_{8}$ & & $\|_{3495.685}$ & $|28598.55|$ & 25 & $50 \mathrm{r}, \mathrm{II}$ & $\left|b^{4} \mathrm{~F}_{2}-b^{4} \mathrm{G}_{3}\right|$ & $\mathrm{S}$ \\
\hline
\end{tabular}


TABLE 3.-Underwater spark absorption spectrum of cobalt-Continued

\begin{tabular}{|c|c|c|c|c|c|c|c|c|c|c|c|}
\hline \multirow{2}{*}{$\lambda$} & \multirow{2}{*}{$\nu$} & \multicolumn{2}{|c|}{$\begin{array}{l}\text { Intensities } \\
\text { and class }\end{array}$} & \multirow{2}{*}{$\begin{array}{c}\text { Combina- } \\
\text { tion }\end{array}$} & \multirow{2}{*}{ Notes } & \multirow{2}{*}{$\lambda$} & \multirow{2}{*}{$\nu$} & \multicolumn{2}{|c|}{$\begin{array}{l}\text { Intensities } \\
\text { and class }\end{array}$} & \multirow{2}{*}{$\underset{\text { tion }}{\text { Combina- }}$} & \multirow{2}{*}{ Notes } \\
\hline & & A & $\mathbf{E}$ & & & & & $\mathbf{A}$ & $\mathbf{E}$ & & \\
\hline 3496.682 & 28590.39 & 10 & $15, \mathrm{I}$ & $b^{4} \mathrm{~F}_{1}-a^{2} \mathrm{G}_{4}$ & & 3602.081 & 27753.85 & 15 & $40 \mathrm{R}, \mathrm{II}$ & $a^{4} \mathrm{~F}_{2}-a^{4} \overline{\mathrm{F}}_{2}$ & $\mathbf{S}$ \\
\hline 3502.281 & \begin{tabular}{|l|}
544.68 \\
\end{tabular} & 75 & $|100 \mathrm{R}, \mathrm{II}|$ & $b^{4} \mathrm{~F}_{5}-b^{4} \mathrm{D}_{4}$ & $\mathrm{~A}, \mathrm{~S}$ & 05.367 & 728.55 & 10 & $20 \mathrm{r}, \mathrm{I}$ & $b^{4} \mathbf{F}_{4}-a^{2} \bar{F}_{4}$ & \\
\hline 06.315 & 511.84 & 50 & $80 \mathrm{R}, \mathrm{II}$ & $b^{4} \mathrm{~F}_{4}-b^{4} \mathrm{D}_{3}$ & $\mathrm{~S}$ & 27.807 & 557.04 & 15 & $25 \mathrm{r}, \mathrm{I}$ & $b^{4} \mathrm{~F}_{4}-a^{2} \mathrm{G}_{5}$ & 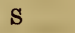 \\
\hline 09.844 & 483.18 & 25 & $50 \mathrm{r}, \mathrm{II}$ & $b^{4} \mathrm{~F}_{3}-b^{4} \mathrm{G}_{4}$ & $\mathbf{S}$ & 31.340 & 530.22 & 10 & $20 \mathrm{r}, \mathrm{I}$ & $a^{4} \mathrm{~F}_{4}-a^{4} \overline{\mathrm{F}}_{5}$ & S \\
\hline 10.419 & 478.51 & 20 & $30 \mathrm{r}, \mathrm{I}$ & $a^{4} \mathrm{~F}_{1}-a^{4} \mathrm{D}_{4}$ & $A, S$ & 47.663 & 407.04 & 5 & $12, \mathrm{I}$ & $a^{4} \mathrm{~F}_{2}-a^{4} \overline{\mathrm{F}}_{3}$ & \\
\hline 12.643 & 460.48 & 40 & $60 \mathrm{R}, \mathrm{II}$ & $b^{4} \mathrm{~F}_{3}-b^{4} \mathrm{D}_{2}$ & $\mathbf{S}$ & 3652.544 & $|27370.41|$ & 5 & $15, I$ & $a^{4} \mathrm{~F}_{3}-a^{4} \overline{\mathrm{F}}_{4}$ & \\
\hline 13.483 & 453.68 & 30 & $50 \mathrm{R}, \mathrm{II}$ & $a^{4} \mathrm{~F}_{4}-a^{4} \mathrm{G}_{8}$ & S & $\begin{array}{r}3704.061 \\
\end{array}$ & $\mid \begin{array}{r}26989.74 \\
784\end{array}$ & 5 & 25,1 & $a^{2} \mathrm{~F}_{3}-b^{2} \mathrm{~F}_{4}$ & \\
\hline 18. 353 & 414.29 & 20 & $50 \mathrm{R}, \mathrm{II}$ & $a^{2} \mathrm{~F}_{3}-b^{2} \mathrm{D}_{2}$ & $\mathrm{~s}$ & $\begin{array}{r}32.400 \\
3745.501\end{array}$ & $\begin{array}{l}784.82 \\
691.14\end{array}$ & 1 & $20, \mathrm{I}$ & & \\
\hline 20.087 & 400.30 & 10 & $15, \mathrm{II}$ & $a^{4} \mathrm{~F}_{4}-a^{4} \overline{\mathrm{F}}_{3}$ & $\mathrm{~s}$ & 3745.501 & $\begin{array}{r}691.14 \\
\end{array}$ & औ) & $25, \mathrm{I}$ & $a^{2} \mathrm{~F}_{4}-b^{2} \mathrm{G}_{4}$ & $\mathrm{~S}$ \\
\hline 21.572 & 388.32 & 15 & $30 \mathrm{r}, \mathrm{I}$ & $b^{4} F_{5}-a^{2} \bar{F}_{4}$ & $\mathbf{S}$ & 3842.056 & 526020.38 | & 10 & 30, II & $a^{2} F_{1}-a^{2} D_{3}$ & \\
\hline & & & & & & 45. 474 & 25997.25 & 50 & $60, \mathrm{II}$ & $a^{2} \mathrm{~F}_{4}-b^{2} \mathrm{G}_{5}$ & $\mathrm{~s}$ \\
\hline 23.438 & 373.28 & 20 & $25 \mathrm{r}, \mathrm{I}$ & $b^{4} F_{2}-b^{4} D_{1}$ & $\mathrm{~S}$ & 61.168 & 3 891.59 & $2 \mathrm{p}$ & $20, \mathrm{I}$ & $a^{2} \mathrm{~F}_{3}-a^{2} \mathrm{D}_{2}$ & \\
\hline 26. 856 & 345.79 & 50 & $100 \mathrm{R}, \mathrm{I}$ & $a^{4} \mathrm{~F}_{5}-a^{4} \overline{\mathrm{F}}_{5}$ & $\mathrm{~A}, \mathrm{~S}$ & 73. 117 & 811.71 & 30 & $60, \mathrm{II}$ & $b^{4} \mathrm{~F}_{5}-a^{4} \mathrm{D}_{4}$ & is \\
\hline 29.037 & 328.27 & 20 & $30 \mathrm{r}, \mathrm{I}$ & $a^{4} \mathrm{~F}_{3}-a^{4} \mathrm{G}_{4}$ & $\mathrm{~s}$ & 73. 957 & 806.11 & 25 & 40, II & $b^{4} \mathrm{~F}_{4}-a^{4} \mathrm{D}_{3}$ & $\mathbf{S}$ \\
\hline 29.815 & 322.03 & 60 & $80 \mathrm{R}, \mathrm{II}$ & $b^{4} \mathrm{~F}_{4}-b^{4} \mathrm{G}_{5}$ & $\mathrm{~A}, \mathrm{~S}$ & 76. 840 & 786. 92 & 1 & $20, I_{1}$ & $b^{4} \mathrm{~F}_{5}-a^{4} \mathrm{G}_{8}$ & $1=$ \\
\hline 33. 363 & 293.60 & 20 & $25 \mathrm{r}, \mathrm{I}$ & $a^{4} \mathrm{~F}_{2}-a^{4} \mathrm{G}_{3}$ & S & $\begin{array}{l}81.877 \\
81.911\end{array}$ & $\left.\begin{array}{l}753.46 \\
753.24\end{array}\right\}$ & 10 & $25, \mathrm{I}$ & $b^{4} \mathrm{~F}_{3}-a^{4} \mathrm{D}_{2}$ & \\
\hline 50.599 & 156.25 & 10 & $20 \mathrm{r}, \mathrm{I}$ & $a^{4} \mathrm{~F}_{3}-a^{4} \overline{\mathrm{F}}_{2}$ & & 94.086 & 672.72 & 30 & 60, II & $a^{2} \mathrm{~F}_{3}-b^{2} \mathrm{G}_{4}$ & $s$ \\
\hline 60.896 & 074.83 & 10 & $20 \mathrm{r}, \mathrm{I}$ & $b^{4} \mathrm{~F}_{2}-b^{4} \mathrm{D}_{2}$ & $\mathrm{~S}$ & 3894.981 & 666.82 & 10 & 20, II & $b^{4} \mathrm{~F}_{2}-a^{4} \mathrm{D}_{1}$ & $\mathrm{~S}$ \\
\hline 64.955 & \begin{tabular}{|l|}
042.87 \\
\end{tabular} & 15 & $25 \mathrm{r}, \mathrm{I}$ & $b^{4} \mathrm{~F}_{3}-a^{2} \mathrm{G}_{4}$ & s & & & & & & \\
\hline 69.382 & 28008.09 & 50 & $80 \mathrm{R}, \mathrm{II}$ & $a^{2} \mathrm{~F}_{4}-b^{4} \bar{F}_{4}$ & $\mathbf{S}$ & 3909.941 & 568.62 & 1 & $15, \mathrm{I}$ & $a^{4} \mathrm{~F}_{5}-a \gamma_{6}$ & $\mathbf{S}$ \\
\hline 74. 964 & 27964.36 & 15 & $25 \mathrm{r}, \mathrm{I}$ & $b^{4} \mathrm{~F}_{3}-b^{4} \mathrm{D}_{3}$ & $\mathrm{~s}$ & \begin{tabular}{|l|}
35.974 \\
95.312
\end{tabular} & $\begin{array}{l}399.51 \\
022.29\end{array}$ & $\begin{array}{r}8 \mathrm{p} \\
25\end{array}$ & $\begin{array}{l}30, \mathrm{II} \\
60, \mathrm{II}\end{array}$ & $\begin{array}{l}a^{2} \mathrm{~F}_{4}-b^{4} \mathrm{~F}_{5} \\
a^{2} \mathrm{~F}_{4}-b^{4} G_{5}\end{array}$ & s \\
\hline 75.361 & 961.25 & 25 & $60 \mathrm{r}, \mathrm{II}$ & $a^{4} \mathrm{~F}_{3}-a^{4} \overline{\mathrm{F}}_{4}$ & S & 3997.909 & 25006.03 & $10 \mathrm{p}$ & $40, \mathrm{II}$ & $a^{2} \mathrm{~F}_{3}-b^{4} \overline{\mathrm{F}}_{4}$ & $\mathbf{S}$ \\
\hline 84.796 & 887.66 & 5 & $15, \mathrm{I}$ & $a^{4} \mathrm{~F}_{3}-a^{4} \mathrm{D}_{4}$ & S & 4092.397 & 24428.69 & $2 \mathrm{p}$ & $25, \mathrm{I}$ & $a^{2} \mathrm{~F}_{4}-a^{2} \overline{\mathrm{F}}_{4}$ & \\
\hline 85.159 & 884.84 & 20 & $25 \mathrm{R}, \mathrm{I}$ & $b^{4} F_{4}-b^{4} D_{4}$ & $\mathrm{~s}$ & 4110.544 & $\begin{array}{l}320.85 \\
\end{array}$ & $1 \mathrm{p}$ & $25, \mathrm{I}$ & $a^{2} \mathrm{~F}_{3}-a^{2} \overline{\mathrm{F}}_{3}$ & \\
\hline 87.188 & 869.06 & 30 & $70 \mathrm{R}, \mathrm{II}$ & $a^{2} \mathrm{~F}_{3}-b^{2} \overline{\mathrm{F}}_{3}$ & & 18. 784 & | 272.18 & $15 \mathrm{p}$ & $50, \mathrm{II}$ & $a^{2} \mathrm{~F}_{3}-a^{2} \mathrm{G}_{4}$ & \\
\hline 3594.869 & 809.52 & 20 & $50 \mathrm{R}, \mathrm{II}$ & $a^{4} \mathrm{~F}_{3}-a^{4} \overline{\mathrm{F}}_{3}$ & $\mathbf{S}$ & 4121.329 & 24257.20 & $30 \mathrm{p}$ & $60, \mathrm{II}$ & $a^{2} \mathrm{~F}_{4}-a^{2} \mathrm{G}_{5}$ & \\
\hline
\end{tabular}

TABLE 4.-Low levels in the spectra of Co

\begin{tabular}{|c|c|c|c|c|c|c|c|c|c|}
\hline $\begin{array}{l}\text { Spec- } \\
\text { trum }\end{array}$ & Value & $\begin{array}{l}\text { Differ- } \\
\text { ence }\end{array}$ & Symbol & Electrons & $\begin{array}{l}\text { Spec- } \\
\text { trum }\end{array}$ & Value & $\begin{array}{l}\text { Differ- } \\
\text { ence }\end{array}$ & Symbol & Electrons \\
\hline Co I & $\begin{array}{r}0.00 \\
815.98 \\
1406.83 \\
1809.30 \\
3482.76 \\
4142.61 \\
4690.10 \\
5075.75 \\
7442.39 \\
8460.77 \\
13795.44 \\
14036.20 \\
14399.15\end{array}$ & $\begin{array}{r}815.98 \\
590.85 \\
402.47 \\
\\
659.85 \\
547.49 \\
385.65 \\
1018.38 \\
240.76 \\
362.95\end{array}$ & $\begin{array}{l}a^{4} \mathrm{~F}_{5} \\
a^{4} \mathrm{~F}_{4} \\
a^{4} \mathrm{~F}_{3} \\
a^{4} \mathrm{~F}_{2} \\
b^{4} \mathrm{~F}_{5} \\
b^{4} \mathrm{~F}_{4} \\
b^{4} \mathrm{~F}_{3} \\
b^{4} \mathrm{~F}_{2} \\
a^{2} \mathrm{~F}_{4} \\
a^{2} \mathrm{~F}_{3} \\
a^{4} \mathrm{P}_{3} \\
a^{4} \mathrm{P}_{2} \\
a^{4} \mathrm{P}_{1}\end{array}$ & $\begin{array}{l}4 s, 3 d^{8} . \\
4 s^{2}, 3 d^{7} \\
4 s^{2}, 3 d^{7}\end{array}$ & c. & $\begin{array}{r}15183.98 \\
15773.94 \\
16195.54 \\
0.0 \\
678.5 \\
1210.4 \\
1590.7 \\
1854.2\end{array}$ & $\begin{array}{l}589.96 \\
421.60 \\
678.5 \\
531.9 \\
389.3 \\
254.5\end{array}$ & $\begin{array}{l}b^{4} \mathrm{P}_{3} \\
b^{4} \mathrm{P}_{3} \\
b^{4} \mathrm{P}_{1} \\
a^{5} \mathrm{~F}_{8} \\
a^{5} \mathrm{~F}_{4} \\
a^{5} \mathrm{~F}_{3} \\
a^{5} \mathrm{~F}_{2} \\
a^{5} \mathrm{~F}_{1} \\
a^{3} \mathrm{~F}_{4} \\
a^{3} \mathrm{~F}_{3} \\
a^{3} \mathrm{~F}_{2}\end{array}$ & $4 s, 3 d r$ \\
\hline
\end{tabular}


3. NICKEL $(\mathrm{Ni}=58.68 ; \mathrm{Z}=28)$

Strips of metal were first cut from nickel anode metal and used as electrodes in obtaining underwater spark spectrograms. This metal was known to contain a small amount of iron, and absorption lines due to this impurity appeared so prominently in the spectrograms that the observations were repeated with electrodes cut from specially purified electrolytic nickel slabs. Two hundred and twenty-five absorption lines appeared on the plates; they are listed in Table 5, in which the wave lengths shorter than $2300 \mathrm{~A}$ are quoted from Piña (29) and the longer one from Hamm (30). The arc emission intensities are from the same sources except for wave lengths greater than 2981 A, where King's (31) intensity estimates and temperature classes are used. The lines identified as absorption lines by Angerer and Joos are noted with the letter $\mathrm{A}$, and a few lines which are identifiable with lines characteristic of the ionized atom are marked $\mathrm{Ni}^{+}$.

Soon after our observations on the absorption spectrum of nickel in underwater sparks were completed we became aware of two similar investigations: Majumder (32) found 88 lines (2254-3858 A) absorbed in the underwater spark, and Narayan and Rao (33) described 180 lines (2121-3858 A) as absorbed. The data were discussed in relation to the published analyses of the structure of the nickel arc spectrum, but in neither case was any addition to this analysis made.

The structure of the nickel arc spectrum has been discussed by Walters (34) and by Bechert and Sommer (35), the term combinations in Table 5 being taken in large part from these papers but with a revision of the notation. The arc spectrum of nickel has three terms which account for all the arc lines identified in absorption; they are ${ }^{3} \mathrm{~F},{ }^{3} \mathrm{D}$, and ${ }^{1} \mathrm{D}$ in the order of increasing energy, but the first two completely overlap and all three have a range of only slightly more than one-third of a volt in the energy scale. The complete set of low terms is presented in table 6 .

Approximately 1,200 lines are observed in the arc emission spectrum of $\mathrm{Ni}$, and nearly 20 per cent of these were absorbed in our underwater spark spectra. A summary of the classified absorption lines shows that 60 with intensity sum 1,160 begin with $a^{3} \mathrm{~F}$, 77 with total intensity 2,410 originate with $a^{3} \overline{\mathrm{D}}$, and 15 with intensity totaling 200 have $a^{1} \mathrm{D}$ as initial state. The levels of $a^{3} \overline{\mathrm{D}}$ are entirely encompassed by those of $a^{3} \mathrm{~F}$, and $a^{3} \mathrm{~F}_{4}$ has the largest quantum numbers as well as the lowest energy level. Nevertheless, the $a^{3} \overline{\mathrm{D}}$ term appears to have considerable advantage over the others in the production of absorption lines.

The spark spectrum of nickel is being analyzed by Dr. A. G. Shenstone, and he has kindly placed at our disposal the relative 
terms which he has found. The low-energy ${ }^{4} \mathrm{~F}$ and ${ }^{2} \mathrm{~F}$ terms are quoted in Table 6; the combinations of these with higher levels account for all of the lines of ionized nickel listed in Table 5. It is probable that $a^{4} \mathrm{~F}$ and $a^{2} \mathrm{~F}$ are low metastable states and that the normal state of $\mathrm{Ni}^{+}$is represented by a doublet $\mathrm{D}$ term arising from nine $d$ electrons. (See Laporte, Zeitschr. f. Phys., 39, p. 123; 1926.) The latter term has not yet been identified; its principal combinations probably gave wave lengths shorter than $2000 \mathrm{~A}$.

TABLE 5.-Underwater spark absorption spectrum of nickel

\begin{tabular}{|c|c|c|c|c|c|c|c|c|c|c|c|}
\hline \multirow{2}{*}{$\lambda$} & \multirow{2}{*}{$\nu$} & \multicolumn{2}{|c|}{$\begin{array}{l}\text { Intensities } \\
\text { and class }\end{array}$} & \multirow{2}{*}{$\underset{\text { tion }}{\text { Combina- }}$} & \multirow{2}{*}{ Notes } & \multirow{2}{*}{$\lambda$} & \multirow{2}{*}{$\nu$} & \multicolumn{2}{|c|}{$\begin{array}{l}\text { Intensities } \\
\text { and class }\end{array}$} & \multirow{2}{*}{$\underset{\text { tion }}{\text { Combina- }}$} & \multirow{2}{*}{ Notes } \\
\hline & & A & $\mathbf{E}$ & & & & & $\mathbf{A}$ & $\mathbf{E}$ & & \\
\hline 2124.84 & 47047.4 & 3 & 2 & & & 2251.52 & 44400.6 & 3 & 2 & $a^{3} \overline{\mathrm{D}}_{2}-\nabla^{\prime}$ & \\
\hline 25.68 & 47028.8 & 5 & 1 & $a^{3} \mathrm{~F}_{4}-4_{3}{ }^{*}$ & & 53.62 & 359.3 & 10 & 2 & $a^{3} \overline{\mathrm{D}}_{3}-c^{3} \overline{\mathrm{F}}_{3}$ & \\
\hline 28.50 & 46966.5 & 3 & $3+$ & & & 53.91 & 353.6 & 10 & 3 & $a^{4} \mathrm{~F}_{2}-{ }^{4} \mathrm{G}_{3}{ }^{*}$ & $\mathrm{Ni}^{+}$ \\
\hline 28.59 & 964.6 & 3 & $3+$ & & & 54.84 & 335.3 & 8 & 2 & & \\
\hline 29.96 & 934.4 & 10 & $0+$ & $a^{3} \overline{\mathrm{D}}_{2}-3_{2}{ }^{*}$ & & 55.89 & 314.6 & 2 & 2 & $a^{3} \mathrm{~F}_{4}-r_{4}^{\prime}$ & \\
\hline 34.95 & 824.7 & 20 & 2 & $a^{3} \overline{\mathrm{D}}_{3}-43^{*}$ & & 58.16 & 270.1 & 6 & 3 & $a^{3} \overline{\mathrm{D}}_{3}-\mathrm{t}_{2}{ }_{2}$ & 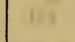 \\
\hline 47.78 & 545.0 & 40 & 2 & & & 59.56 & 242.7 & 7 & 3 & $a^{3} \overline{\mathrm{D}}_{1}-1_{1} *$ & 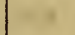 \\
\hline 51.94 & 455.0 & 3 & 1 & & 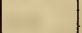 & 61.43 & 206.1 & 10 & $2+$ & $a^{3} F_{4}-q_{3}^{\prime}$ & \\
\hline 52.22 & 449.0 & & 1 & & & $64.5 \mathrm{C}$ & 146.2 & 3 & 3 & $a^{4} \mathrm{~F}_{3}-{ }^{4} \mathrm{G}_{4} *$ & $\mathrm{Ni}+$ \\
\hline 57.84 & 328.0 & 10 & 2 & - & & 66.40 & 109.2 & 3 & 2 & $a^{3} \overline{\mathrm{D}}_{3}-\mathbf{r}_{4}^{\prime}{ }^{*}$ & \\
\hline 58.34 & 317.3 & 30 & 3 & $a^{3} \overline{\mathrm{D}}_{3}-22^{*}$ & & 67.61 & 085.6 & 2 & 2 & $a^{3} \bar{F}_{3}-c^{3} \bar{F}_{2}$ & \\
\hline 61.08 & 258.6 & & 2 & $a^{3} \overline{\bar{D}}_{2}-3{ }^{*}$ & & 70.30 & 033.4 & & 3 & $a^{4} \mathrm{~F}_{4}-{ }^{4} \mathrm{G}_{5}^{*}$ & $\mathrm{Ni}^{+}$ \\
\hline 61.25 & 254.9 & 6 & 2 & & & 70.47 & 030.1 & 5 & 3 & & \\
\hline 65.55 & 163.1 & 5 & 3 & $a^{4} \mathrm{~F}_{5}-4 \overline{\mathrm{F}} 5^{*}$ & $\mathrm{Ni}^{+}$ & 71.96 & 44001.2 & 6 & 2 & $a^{3} \overline{\mathrm{D}}_{3}-\mathrm{q}_{3}^{\prime}$ & \\
\hline 66.14 & 150.5 & 5 & $1+$ & $a^{3} \overline{\mathrm{D}_{2}}-4^{*}$ & & 77.78 & 43888.8 & 2 & $1+$ & & \\
\hline 69.05 & 46088.6 & 3 & 3 & $a^{4} \mathrm{~F}_{4}-4 \overline{\mathrm{F}}_{4}^{*}$ & $\mathrm{Ni}^{+}$ & 78.79 & 869.4 & 3 & $2+$ & $a^{2} \mathrm{~F}_{4}-{ }^{2} \mathrm{D}_{3} *$ & $\mathrm{Ni}^{+}$ \\
\hline 74.44 & 45974.4 & 10 & 2 & & & 87.12 & 709.6 & & 2 & $a^{2} \mathrm{~F}_{3}-{ }^{2} \mathrm{D}_{2}$ & $\mathrm{Ni}^{+}$ \\
\hline 74.64 & 970.2 & 10 & 3 & $a^{4} \mathrm{~F}_{4}-{ }^{2} \mathrm{G}_{3}$ & $\mathrm{Ni}^{+}$ & 87.40 & 704.2 & 8 & 2 & $a^{3} \overline{\mathrm{D}}_{1}-c^{3} \overline{\mathrm{F}}_{2}$ & \\
\hline 75.11 & 960.2 & 2 & 3 & $a^{4} \mathrm{~F}_{3}-{ }^{4} \overline{\mathrm{F}}_{3}{ }^{*}$ & $\mathrm{Ni}^{+}$ & 88.46 & 684.0 & 4 & 3 & $a^{3} \overline{\mathrm{D}}_{2}-c^{3} \overline{\mathrm{F}}_{3}$ & \\
\hline 82.37 & 807.3 & 7 & 2 & $a^{3} F_{3}-32^{*}$ & & 90.08 & 653.1 & 25 & 6 & $a^{3} F_{4}-0^{\prime}{ }_{3}$ & \\
\hline 83.28 & 788.3 & 2 & 3 & & . & 93.15 & 594.7 & 5 & 3 & $a^{3} \overline{\mathrm{D}}_{2}-\mathrm{t}_{2}{ }_{2}$ & \\
\hline 83.73 & 778.8 & & 3 & & & 96.54 & 530.3 & 2 & 3 & $a^{2} F_{4}-2 \bar{F}_{4}^{*}$ & $\mathrm{Ni}^{+}$ \\
\hline 83.94 & 774.4 & 2 & 3 & & & 97.53 & 511.6 & 2 & 3 & $a^{4} \mathrm{~F}_{2}-{ }^{4} \mathrm{D}_{1}$ & $\mathrm{Ni}^{+}$ \\
\hline 84.55 & 761.6 & 2 & 3 & $a^{4} \mathrm{~F}_{2}-{ }^{4} \overline{\mathrm{F}}_{2} *$ & $\mathrm{Ni}^{+}$ & 2298.26 & 497.7 & 4 & 3 & $a^{2} \bar{F}_{3}-2 \bar{F}_{3}^{*}$ & $\mathrm{Ni}^{+}$ \\
\hline 90.24 & 642.8 & 15 & 2 & $a^{3} \overline{\mathrm{D}}_{3}-22^{*}$ & & 2300.773 & 450.25 & 20 & $2 \mathrm{u}$ & $a^{3} \overline{\mathrm{D}}_{3}-0^{\prime} 3$ & \\
\hline 2197. 38 & 494.5 & 20 & 3 & & & 02.973 & 408.76 & 10 & 3 & $\left\{\begin{array}{l}a^{3} \overline{\mathrm{D}}_{1}-\mathrm{l}_{1}^{*} \\
a^{4}\end{array}\right.$ & \\
\hline 2200.72 & 425.4 & 4 & 2 & $a^{3} \overline{\mathrm{D}_{1}}-32^{*}$ & & & & & & $\left(a^{4} \mathrm{~F}_{4}-{ }^{4} \mathrm{D}_{3}{ }^{*}\right.$ & $\mathrm{Ni}^{+}$ \\
\hline 01.40 & 411.4 & 8 & 3 & $a^{4} \mathrm{~F}_{2}-{ }^{4} \overline{\mathrm{F}}_{3} *$ & $\mathrm{Ni}^{+}$ & 07.353 & $\begin{array}{l}326.33 \\
\end{array}$ & 3 & 1 & & \\
\hline 01.60 & 407.3 & 8 & 2 & & & $\begin{array}{l}09.486 \\
10.025\end{array}$ & $\begin{array}{l}286.33 \\
276.24\end{array} \mid$ & 100 & $\begin{array}{l}1 \\
1\end{array}$ & & \\
\hline 06.75 & 301.3 & 3 & $3]$ & $a^{4} \mathrm{~F}_{3}-4 \bar{F}_{4} *$ & $\mathrm{Ni}^{+}$ & $\begin{array}{l}10.025 \\
10.955\end{array}$ & $\begin{array}{l}276.24 \\
258.83\end{array}$ & & $3 \mathrm{R}$ & $a^{3} \mathbf{F}_{4}-c^{3} \bar{F}_{4}$ & A \\
\hline 11.32 & 207.7 & 2 & 2 & & & 12. 338 & 232.95 & 50 & $3 \mathrm{R}$ & $a^{3} \mathrm{~F}_{3}-c^{3} \overline{\mathrm{F}}_{3}$ & $\mathbf{A}$ \\
\hline 12.17 & 190.3 & 2 & 2 & $a^{3} \mathrm{~F}_{3}-22^{*}$ & & 13. 656 & 208. 33 & $\operatorname{lom}$ & $2 \mathrm{u}$ & & $\mathbf{A}$ \\
\hline 16. 48 & 102.5 & 9 & 3 & $a^{4} \mathrm{~F}_{5}-{ }^{4} \mathrm{G}_{6} *$ & $\mathrm{Ni}^{+}$ & 13.982 & $202.24]$ & \}$^{100}$ & $2 \mathrm{u}$ & $a^{3} \mathrm{~F}_{2}-c^{3} \overline{\mathrm{F}}_{2}$ & \\
\hline $16.65^{\circ}$ & $\begin{array}{l}099.0 \\
\end{array}$ & & 2 & & & 16.040 & 163.86 & 10 & 2 & $a^{4} F_{5}-{ }^{4} D_{4}^{*}$ & $\mathrm{Ni}^{+}$ \\
\hline 17.82 & 45075.2 & 2 & 2 & & & 17.158 & 143.03 & 50 & $2 \mathrm{R}$ & $a^{3} F_{3}-t^{\prime}{ }_{2}$ & A \\
\hline 22.08 & 44988.8 & 5 & 2 & & & 20.034 & 089.55 & 100 & $5 \mathrm{R}$ & $a^{3} \mathrm{~F}_{4}-\mathrm{n}_{5}^{\prime}$ & A \\
\hline 23.08 & 968.6 & 2 & 3 & $a^{4} \mathrm{~F}_{5}-4 \mathrm{G}_{5}^{*}$ & $\mathrm{Ni}^{+}$ & 21.387 & 43064.44 & 60 & $3 \mathrm{R}$ & $a^{3} F_{2}-\nabla^{\prime}$ & H \\
\hline 24.89 & 932.0 & 3 & $2+$ & $a^{4} \mathrm{~F}_{4}-{ }^{4} \mathrm{G}_{4} *$ & $\mathrm{Ni}^{+}$ & 25. 799 & 42982.76 & 50 & $3 \mathrm{R}$ & $a^{3} F_{3}-r_{4}^{\prime}$ & A. \\
\hline 31.03 & 808.4 & 3 & 2 & $a^{3} \overline{\mathrm{D}}_{1}-22^{*}$ & & 29.974 & 905.74 & 50 & 2 & $a^{3} \mathrm{~F}_{2}-\mathrm{l}_{1}{ }^{*}$ & \\
\hline 2244.51 & 44539.3 & 3 & 3 & $a^{3} \bar{D}_{2}-c^{3} \bar{F}_{2}$ & & 2331.704 & 42873.92 & 2 & 1 & $a^{3} F_{3}-q^{\prime} 3$ & \\
\hline
\end{tabular}


TABLE 5.-Underwater spark absorption spectrum of nickel-Continued

\begin{tabular}{|c|c|c|c|c|c|c|c|c|c|c|c|}
\hline \multirow{2}{*}{$\lambda$} & \multirow{2}{*}{$\nu$} & \multicolumn{2}{|c|}{$\begin{array}{l}\text { Intensities } \\
\text { and class }\end{array}$} & \multirow{2}{*}{$\begin{array}{c}\text { Combina- } \\
\text { tion }\end{array}$} & \multirow{2}{*}{ Notes } & \multirow{2}{*}{$\lambda$} & \multirow{2}{*}{$\nu$} & \multicolumn{2}{|c|}{$\begin{array}{l}\text { Intensities } \\
\text { and class }\end{array}$} & \multirow{2}{*}{$\underset{\text { tion }}{\text { Combina- }}$} & \multirow{2}{*}{ Notes } \\
\hline & & A & $\mathbf{E}$ & & & & & A & $\mathbf{E}$ & & \\
\hline 2337.097 & $42774.99 \mid$ & 50 & 1 & $a^{3} \overline{\mathrm{D}}_{2}-0^{\prime}{ }_{3}$ & & 2510.885 & 39814.61 & 5 & 5 & $a^{2} \mathrm{~F}_{4}-{ }^{4} \mathrm{G}_{5} *$ & $\mathrm{Ni}^{+}$ \\
\hline 37.488 & 767.83 & 50 & 1 & $a^{3} \mathbf{F}_{4}-l^{\prime}{ }_{3}$ & A & 24.221 & 604.27 & 5 & 1 & $a^{3} \overline{\mathrm{D}}_{2}-\mathrm{b}_{2}^{\prime}$ & \\
\hline 38.500 & 749.33 & 2 & 1 & $a^{3} \overline{\mathbf{D}}_{3}-\mathrm{m}_{2}^{\prime}$ & & 40.027 & 357.84 & $1 ?$ & 1 & $a^{1} \overline{-}_{2}-l_{3}{ }_{3}$ & \\
\hline 45.545 & 620.94 & 30 & $2 \mathrm{R}$ & $a^{3} \mathrm{~F}_{4}-\mathrm{e}_{4}^{\prime}$ & A & 2545.927 & 39266.64 & 2 & 1 & $a^{2} \mathrm{~F}_{3}-{ }^{4} \mathrm{G}_{4} *$ & $\mathrm{Ni}^{+}$ \\
\hline 46.635 & 601.14 & 4 & & $a^{3} \mathrm{~F}_{3}-\mathrm{u}^{\prime}$ & & 2746.745 & 36395.98 & 5 & & $a^{3} \overline{\mathrm{D}}_{3}-b^{1} \mathrm{D}_{2}$ & \\
\hline 47.532 & 584.87 & 15 & 1 & $a^{3} F_{4}-c^{\prime}{ }_{4}$ & A & 2798. 651: & 35720.98 & 10 & 4 & $a^{3} \overline{\mathrm{D}}_{2}-b^{1} \mathrm{D}_{2}$ & \\
\hline 48. 738 & 563.00 & 2 & 1 & $a^{3} \overline{\mathrm{D}}_{3}-\mathrm{l}_{3}{ }_{3}$ & & 2821.296 & 35434.30 & 15 & 4. & $a^{3} \bar{D}_{3}-b^{1} \overline{\mathrm{F}}_{3}$ & \\
\hline 55.060 & 448. 76 & 10 & 1 & $a^{3} \overline{\mathrm{D}}_{3}-\mathrm{k}_{2}^{\prime}$ & & 2865.508 & 34887.61 & 1 & 5 & $a^{3} \overline{\mathrm{D}}_{1}-b^{1} \mathrm{D}_{2}$ & \\
\hline 56.869 & 416. 18 & 10 & 1 & $a^{3} \overline{\mathrm{D}}_{3}-\mathrm{e}_{4}^{\prime}$ & & 2943.922 & 33958.39 & 25 & 6 & $a^{3} \overline{\mathrm{D}}_{3}-b^{3} \mathrm{D}_{2}$ & \\
\hline 58.865 & 380.29 & 8 & 1 & $a^{3} \overline{\mathrm{D}}_{3}-c^{\prime}{ }_{4}$ & & 81.652 & 528.69 & 20 & $20 \mathrm{R}, \mathrm{II}$ & $a^{3} \overline{\mathrm{D}}_{2}-b^{3} \mathrm{D}_{1}$ & \\
\hline 60.637 & 348.48 & 10 & 1 & $a^{3} F_{2}-c^{3} \overline{F_{8}}$ & & 84.129 & 500.87 & 10 & $12 \mathrm{R}, \mathrm{II}$ & $a^{3} \mathbf{F}_{4}-b^{3} D_{3}$ & \\
\hline 62.061 & 322.96 & 10 & 1 & $a^{3} F_{3}-o^{\prime}{ }_{3}$ & & 92.597 & 406.08 & 15 & $20 \mathrm{R}, \mathrm{II}$ & $a^{3} \overline{\mathrm{D}}_{3}-b^{3} \overline{\mathrm{F}}_{2}$ & \\
\hline 65.677 & 258.27 & 1 & 1 & $a^{3} \mathrm{~F}_{2}-\mathrm{t}^{\prime}{ }_{3}$ & & 2994.458 & 385.31 & 20 & $25 \mathrm{R}, \mathrm{II}$ & $a^{3} \overline{\mathrm{D}}_{3}-a^{1} \mathrm{G}_{4}$ & \\
\hline 76.019 & 42074.35 & 7 & 1 & $a^{3} \overline{\mathbf{D}}_{2}-\mathrm{m}_{2}^{\prime}$ & & 3002.492 & 295.98 & 80 & $100 \mathrm{R}, \mathrm{II}$ & $a^{3} \bar{D}_{3}-b^{3} D_{3}$ & \\
\hline 80.787 & 41990.09 & 2 & 1 & $a^{3} F_{2}-q^{\prime} 3$ & & 03.628 & 283.40 & 40 & $60 \mathrm{R}, \mathrm{II}$ & $a^{3} \overline{\mathrm{D}}_{2}-b^{3} \mathrm{D}_{2}$ & \\
\hline 84.400 & 926. 47 & 6 & 1 & $a^{3} \bar{F}_{3}-c^{3} \bar{F}_{4}$ & & 12.007 & 190.81 & 60 & $75 \mathrm{R}, \mathrm{II}$ & $a^{1} \overline{\mathrm{D}}_{2}-b^{1} \mathrm{D}_{2}$ & \\
\hline 85.015 & 915.66 & 3. & 1 & & & 19.150 & 33112.29 & 20 & $20 \mathrm{R}, \mathrm{II}$ & $a^{3} \bar{F}_{4}-b^{3} \bar{F}_{3}$ & \\
\hline 86.587 & 888.06 & 10 & 1 & $a^{3} \overline{\mathrm{D}}_{2}-\mathrm{l}_{3}{ }_{3}$ & & 31.869 & 32973.38 & 10 & $10 \mathrm{r}, \mathrm{II}$ & $a^{3} \mathrm{~F}_{4}-b^{3} \overline{\mathrm{F}}_{4}$ & \\
\hline 87.556 & 871.05 & 4 & 1 & & & 37.940 & 907.49 & 60 & $60 \mathrm{R}, \mathrm{II}$ & $a^{3} \overline{\mathrm{D}}_{3}-b^{3} \overline{\mathrm{F}}_{3}$ & \\
\hline 92.963 & $776.46\}$ & & 1 & & & 45.012 & 831.07 & 5 & $10 \mathrm{r}, \mathrm{II}$ & $a^{3} F_{3}-b^{3} D_{2}$ & \\
\hline 93.115 & $773.80\}$ & 15 & 1 & $a^{3} \overline{\mathrm{D}}_{2}-\mathrm{k}_{2}^{\prime}$ & & 50.828 & 768.49 & 100 & $100 \mathrm{R}, \mathrm{II}$ & $a^{3} \overline{\mathrm{D}}_{3}-b^{3} \overline{\mathrm{F}}_{4}$ & \\
\hline 94.523 & 749.24 & 10 & 2 & $a^{2} F_{4}-{ }^{2} G_{5} *$ & $\mathrm{Ni}^{+}$ & 54.317 & 731.06 & 40 & $50 \mathrm{R}, \mathrm{II}$ & $a^{3} \overline{\mathrm{D}}_{2}-b^{3} \overline{\mathrm{F}}_{2}$ & \\
\hline 96.385 & 716.80 & 3 & 1 & $a^{3} \mathrm{~F}_{2}-\mathrm{u}^{\prime}$ & & 57.647 & 695.41 & 50 & $50 \mathrm{R}, \mathrm{II}$ & $a^{3} \overline{\mathrm{D}}_{1}-b^{3} \mathrm{D}_{1}$ & \\
\hline 2396.637 & 712.42 & 3 & 1 & $a^{1} \overline{\mathrm{D}}_{2}-\mathrm{l}_{1}^{*}$ & & 64.626 & 620.95 & 20 & $25 \mathrm{R}, \mathrm{II}$ & $a^{3} \underline{\bar{D}}_{2}-b^{3} \mathrm{D}_{3}$ & \\
\hline & & & & & & 80.758 & 450.15 & 10 & $20 \mathrm{R}, \mathrm{II}$ & $a^{3} \bar{D}_{1}-b^{3} D_{2}$ & \\
\hline 2401.846 & 621.97 & 20 & 1 & $a^{3} \mathrm{~F}_{3}-\mathrm{m}_{2}^{\prime}$ & & & & & & & \\
\hline 12.647 & 435.64 & 10 & 1 & $a^{3} \mathrm{~F}_{3}-\mathrm{l}_{3}^{\prime}$ & & 97.120 & 278.72 & 6 & $15 \mathrm{r}, \mathrm{II}$ & $a^{3} \mathrm{~F}_{3}-b^{3} \overline{\mathrm{F}}_{2}$ & \\
\hline 16.140 & 375.74 & 6 & $\omega$ & $a^{2} \mathrm{~F}_{3}-{ }^{2} \mathrm{G}_{4} *$ & $\mathrm{Ni}^{+}$ & 3099.117 & 257.92 & 4 & $12 \mathrm{r}, \mathrm{II}$ & $a^{3} \mathrm{~F}_{3}-a^{1} \mathrm{G}_{4}$ & \\
\hline 19.310 & 321.54 & 20 & 1 & $a^{3} \mathrm{~F}_{3}-\mathrm{k}_{2}^{\prime}$ & & 3101.563 & 232.49 & 125 & $100 \mathrm{R}, \mathrm{II}$ & $a^{3} \overline{\mathrm{D}}_{2}-b^{3} \overline{\mathrm{F}}_{3}$ & \\
\hline 21.231 & 288. 76 & 7 & $2-$ & $a^{3} F_{3}-e_{4}^{\prime}$ & & 01.881 & 229.18 & & $40 \mathrm{R}, \mathrm{II}$ & $a^{1} \overline{\mathrm{D}}_{2}-b^{1} \overline{\mathrm{F}}_{3}$ & \\
\hline & & & 118 & & & 05.466 & 191.98 & 5 & $15 \mathrm{r}, \mathrm{II}$ & $a^{3} \mathrm{~F}_{2}-b^{3} \mathrm{D}_{1}$ & \\
\hline 23.330 & 253.00 & 4 & 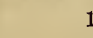 & $a^{3} F_{3}-c_{4}^{\prime}$ & & & & & & & \\
\hline 23.661 & 247.36 & 4 & 1 & & & 14. 128 & 32102.44 & 2 & $20 \mathrm{R}$, II & $a^{3} \mathrm{D}_{2}-a^{1} \mathrm{P}_{1}$ & \\
\hline 24.025 & 241.17 & 5 & 1 & $a^{3} \bar{D}_{1}-m^{\prime}{ }_{2}$ & & 34.106 & 31897.81 & 50 & $60 \mathrm{R}, \mathrm{II}$ & $a^{3} \overline{\mathrm{D}}_{1}-b^{3} \overline{\mathrm{F}}_{2}$ & \\
\hline 32.222 & 102.19 & 2 & 1 & & & 45. 707 & 780.18 & $1 ?$ & $8, \mathrm{II}$ & $a^{3} \overline{\mathrm{F}}_{3}-b^{3} \overline{\mathrm{F}}_{3}$ & \\
\hline 34.431 & 41064.89 & 2 & 1 & $a^{1} \overline{\mathrm{D}}_{2}-\mathrm{t}_{2}^{\prime}$ & & 3197.121 & 269.13 & 5 & $10 \mathrm{r}, \mathrm{II}$ & $a^{3} \overline{\mathrm{D}}_{1}-a^{1} \overline{\mathrm{P}}_{1}$ & \\
\hline & & & & & & 3221. 661: & 31030.96 & 10 & $10 \mathrm{r}, \mathrm{II}$ & $a^{3} \bar{F}_{4}-a^{1} \bar{F}_{3}$ & \\
\hline 41.832 & 40940.44 & 10 & 2 & $a^{3} \overline{\mathrm{D}}_{1}-\mathrm{k}_{2}^{\prime}$ & & 25.030 & 30998.54 & 10 & $10 \mathrm{r}, \mathrm{II}$ & $a^{3} \overline{\mathrm{D}}_{2}-b^{3} \mathrm{D}_{1}$ & \\
\hline 53.997 & 737.51 & 4 & 2 & $a^{3} \mathrm{~F}_{2}-\mathrm{m}_{2}^{\prime}$ & & 26.992 & $\begin{array}{r}979.69 \\
\end{array}$ & 3 & $\begin{array}{r}10 \mathrm{I}, \mathrm{II} \\
5, \mathrm{II}\end{array}$ & $a^{3} \mathrm{~F}_{4}-a^{3} \mathrm{G}_{4}$ & \\
\hline 65.279 & 551.09 & 2 & 1 & $a^{3} \mathrm{~F}_{2}-\mathrm{l}_{3}{ }_{3}$ & & 32.945 & 922.65 & 25 & $25 \mathrm{R}, \mathrm{II}$ & $a^{3} \mathrm{~F}_{4}-a^{3} \mathrm{G}_{5}$ & \\
\hline 72.074 & 439.64 & 6 & 3 & & & 34.658 & 906.28 & 10 & $10 \mathrm{r}, \mathrm{II}$ & $a^{3} \overline{\mathrm{D}}_{2}-a^{3} \mathrm{G}_{2}$ & \\
\hline 72.917 & 425.85 & 4 & 1 & & & 43.064 & 826.18 & 20 & $25 \mathrm{R}, \mathrm{I}$ & $a^{3} \overline{\mathrm{D}}_{3}-a^{1} \overline{\mathrm{F}}_{3}$ & \\
\hline 76.876 & 361.24 & 3 & 2 & $a^{3} F_{4}-a_{3}^{\prime}$ & & 48.43 & 775.2 & & $8, \mathrm{II}$ & $a^{3} \overline{\mathrm{D}}_{3}-a^{3} \mathrm{G}_{4}$ & \\
\hline 79. 489 & 318.71 & 3 & 1 & & & 50.749 & 753.30 & 2 & $9, \mathrm{II}$ & $a^{1} \overline{\mathrm{D}}_{2}-b^{3} \mathrm{D}_{2}$ & \\
\hline 79. 773 & $314.09]$ & & 1 & & & 71.118 & 561.81 & 2 & $10, \mathrm{II}$ & $a^{3} \overline{\mathrm{D}}_{2}-a^{1} \mathrm{D}_{2}$ & \\
\hline 83.285 & 257.08 & 10 & 1 & & & 82.701 & 453.98 & 2 & $8, \mathrm{II}$ & $a^{3} F_{3}-a^{3} G_{3}$ & \\
\hline 84.039 & 244.87 & 5 & 1 & $a^{1} \overline{\mathrm{D}}_{2}-0^{\prime}{ }_{3}$ & & 3286.953 & 414.58 & $1 ?$ & $8, \mathrm{II}$ & $a^{3} \overline{\mathrm{D}}_{3}-1 a^{3} \overline{\mathrm{F}}_{2}$ & \\
\hline 88.149 & 178.39 & 6 & 1 & & & 3315.668 & 151.19 & 30 & $30 \mathrm{R}, \mathrm{II}$ & $a^{3} \overline{\mathrm{D}}_{2}-a^{1} \overline{\mathrm{F}}_{3}$ & \\
\hline 89.51 & 156.4 & 1 & & $a^{3} \overline{\mathrm{D}}_{3}-\mathrm{a}_{3}{ }_{3}$ & & 20.259 & 109.50 & 20 & $20 \mathrm{R}, \mathrm{II}$ & $a^{3} \mathrm{~F}_{3}-a^{1} \mathrm{D}_{2}$ & \\
\hline 90.689 & 137.42 & 4 & 1 & & & 22.316 & 30090.86 & 10 & $15 \mathrm{r}, \mathrm{II}$ & $a^{1} \overline{\mathrm{D}}_{2}-b^{3} \mathrm{D}_{3}$ & \\
\hline 2491.184 & 40129.45 & 4 & 1 & & & 61.557 & 29739.60 & 20 & $20 \mathrm{R}, \mathrm{II}$ & $a^{3} \overline{\mathrm{D}}_{2}-1 a^{3} \overline{\mathrm{F}}_{2}$ & \\
\hline 2501.28 & $|39969,91|$ & $3 !$ & 1 & 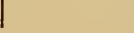 & & 3365.771 & $|29702,37|$ & 10 & $15 \mathrm{r}, \mathrm{II}$ & $a^{1} \overline{\mathrm{D}}_{2}-b^{3} \overline{\mathrm{F}}_{3}$ & \\
\hline
\end{tabular}


TABLE 5.-Underwater spark absorption spectrum of nickel-Continued

\begin{tabular}{|c|c|c|c|c|c|c|c|c|c|c|c|}
\hline \multirow{2}{*}{$\lambda$} & \multirow{2}{*}{$\nu$} & \multicolumn{2}{|c|}{$\begin{array}{l}\text { Intensities } \\
\text { and class }\end{array}$} & \multirow{2}{*}{$\underset{\text { tion }}{\text { Combina- }}$} & \multirow{2}{*}{ Notes } & \multirow{2}{*}{$\lambda$} & \multirow{2}{*}{$\nu$} & \multicolumn{2}{|c|}{$\begin{array}{l}\text { Intensities } \\
\text { and class }\end{array}$} & \multirow{2}{*}{$\underset{\text { tion }}{\text { Combina- }}$} & \multirow{2}{*}{ Notes } \\
\hline & & $\mathbf{A}$ & $\mathbf{E}$ & & & & & $\mathbf{A}$ & $\mathbf{E}$ & & \\
\hline 3366.159 & 29698.86 & 20 & $20 \mathrm{R}, \mathrm{II}$ & $a^{3} \mathrm{~F}_{3}-a^{1} \overline{\mathrm{F}}_{3}$ & & 3500.852 & 28556.33 & 20 & $25 \mathrm{R}, \mathrm{II}$ & $a^{3} F_{3}-a^{3} D_{2}$ & \\
\hline 69.576 & 668.83 & 50 & $80 \mathrm{R}, \mathrm{II}$ & $a^{3} \mathrm{~F}_{4}-a^{3} \mathrm{D}_{3}$ & $\mathbf{A}$ & 02.604 & 542.05 & $1 ?$ & $8, I$ & & \\
\hline 71.995 & 647.55 & 15 & $15 \mathrm{r}, \mathrm{II}$ & & & 10.340 & 479.15 & 50 & $80 \mathrm{R}, \mathrm{II}$ & $a^{3} \overline{\mathrm{D}}_{1}-a^{3} \overline{\mathrm{P}}_{0}$ & \\
\hline 74.228 & 627.93 & 10 & $15 \mathrm{r}, \mathrm{II}$ & $a^{3} \overline{\mathrm{D}}_{3}-a^{3} \overline{\mathrm{F}}_{3}$ & & 15.057 & 440.93 & 125 & $150 \mathrm{R}, \mathrm{II}$ & $a^{3} \overline{\mathrm{D}}_{2}-1 a^{3} \overline{\mathrm{F}}_{3}$ & $\mathbf{A}$ \\
\hline 80.577 & 572.28 & 88 & $80 \mathrm{R}, \mathrm{II}$ & $a^{1} \overline{\mathrm{D}}_{2}-a^{1} \overline{\mathrm{P}}_{1}$ & & 19. 776 & 402.81 & 10 & $20 \mathrm{R}, \mathrm{II}$ & $a^{3} \mathrm{~F}_{2}-1 a^{3} \overline{\mathrm{F}}_{2}$ & \\
\hline 80.885 & 569.59 & & $15 \mathrm{r}, \mathrm{II}$ & & & 24.543 & 364.39 & 200 & $200 \mathrm{R}, \mathrm{II}$ & $a^{3} \overline{\mathrm{D}}_{3}-a^{3} \overline{\mathrm{P}}_{2}$ & A \\
\hline 91.051 & 480.95 & 25 & $50 \mathrm{R}, \mathrm{II}$ & $a^{3} \mathbf{F}_{4}-1 a^{3} \overline{\mathbf{F}}_{4}$ & & 48. 189 & 175.37 & 5 & $20 \mathrm{r}, \mathrm{II}$ & $a^{3} \overline{\bar{D}} 1-a^{3} D_{2}$ & \\
\hline 3392.993 & 464.08 & 50 & $100 \mathrm{R}, \mathrm{II}$ & $a^{3} \overline{\mathrm{D}}_{3}-a^{3} \mathrm{D}_{3}$ & & 66.373 & 28031.72 & 60 & $100 \mathrm{R}, \mathrm{II}$ & $a^{1} \overline{\mathrm{D}}_{2}-a^{1} \mathrm{D}_{2}$ & \\
\hline 3409.579 & 320.75 & 2 & $8, \mathrm{II}$ & $a^{3} \mathbf{F}_{4}-1 a^{3} \overline{\mathrm{F}}_{3}$ & & 71.871 & 27988.57 & 40 & $50 \mathrm{R}, \mathrm{II}$ & $a^{3} \bar{F}_{3}-1 a^{3} \bar{F}_{3}$ & \\
\hline 13.478 & 287.26 & 10 & $25 \mathrm{R}, \mathrm{II}$ & $a^{3} \mathbf{F}_{3}-1 a^{3} \overline{\mathbf{F}}_{2}$ & & 3597.699 & 787.64 & 30 & $50 \mathrm{R}, \mathrm{II}$ & $a^{3} \overline{\mathrm{D}}_{1}-a^{3} \overline{\mathrm{P}}_{1}$ & \\
\hline 13.943 & 283.27 & 5 & $12 \mathrm{r}, \mathrm{II}$ & $a^{3} \overline{\mathrm{D}}_{2}-a^{3} \overline{\mathrm{F}}_{2}$ & & 3602.278 & 752.32 & 1 & $15, \mathrm{II}$ & $a^{3} \bar{F}_{3}-a^{3} \bar{F}_{4}$ & \\
\hline 14. 771 & 276.17 & 150 & $150 \mathrm{R}, \mathrm{II}$ & $a^{3} \overline{\mathrm{D}}_{3}-1 a^{3} \overline{\mathrm{F}}_{4} \mid$ & A & 09.312 & 398.24 & 3 & $15, \mathrm{II}$ & $a^{3} \overline{\mathrm{D}}_{2}-a_{\gamma_{3}}$ & \\
\hline 23. 713 & 199. 71 & 50 & $50 \mathrm{R}, \mathrm{II}$ & $a^{3} \overline{\mathrm{D}}_{1}-a^{3} \mathrm{D}_{1}$ & & 10.45 & 689.5 & 25 & $60 \mathrm{R}, \mathrm{II}$ & $a^{3} \overline{\mathrm{D}}_{2}-a^{3} \overline{\mathrm{P}}_{2}$ & \\
\hline 33.565 & 115.93 & 75 & $70 \mathrm{R}, \mathrm{II}$ & $a^{3} \overline{\mathrm{D}}_{3}-1 a^{3} \overline{\mathrm{F}}_{3}$ & A & 12. 732 & 672.02 & 15 & $30 \mathrm{R}, \mathrm{II}$ & $a^{3} \mathrm{~F}_{2}-a^{3} \mathrm{D}_{2}$ & \\
\hline 37.283 & 084.44 & 30 & $30 \mathrm{R}, \mathrm{II}$ & $a^{3} \mathrm{~F}_{4}-a^{3} \overline{\mathrm{F}}_{4}$ & & 19.391 & 621.11 & 100 & $150 \mathrm{R}, \mathrm{II}$ & $a^{1} \overline{\mathrm{D}}_{2}-a^{1} \overline{\mathrm{F}}_{3}$ & \\
\hline 46.263 & 29008.66 & 100 & $100 \mathrm{R}, \mathrm{II}$ & $a^{3} \underline{\mathrm{D}}_{2}-a^{3} \underline{\mathrm{D}}_{2}$ & $\mathbf{A}$ & 3624.733 & 27580.40 & 1 & $15, \mathrm{II}$ & $a^{3} \underline{F}_{4}-a \gamma_{8}$ & \\
\hline 52.891 & 28952.97 & 40 & $40 \mathrm{R}, \mathrm{II}$ & $a^{3} \overline{\mathrm{D}}_{2}-a^{3} \overline{\mathrm{F}}_{3}$ & & 3775.562 & 26478.63 & $3 \mathrm{p}$ & $30 \mathrm{r}, \mathrm{II}$ & $a^{1} \overline{\mathrm{D}}_{2}-a^{3} \mathrm{D}_{2}$ & \\
\hline 58.467 & 906.29 & 100 & $125 \mathrm{R}, \mathrm{II}$ & $a^{3} \overline{\mathrm{D}}_{1}-1 a^{3} \overline{\mathrm{F}}_{2}$ & $\mathbf{A}$ & 3783.521 & 422.93 & $3 \mathrm{p}$ & $30 \mathrm{r}, \mathrm{II}$ & $a^{1} \overline{\mathrm{D}}_{2}-a^{3} \overline{\mathrm{F}} \mathbf{8}$ & \\
\hline 61.660 & 879.63 & 125 & $125 \mathrm{R}, \mathrm{II}$ & $a^{3} \overline{\mathrm{D}}_{3}-a^{3} \overline{\mathrm{F}}_{4}$ & $\mathbf{A}$ & 3807.135 & 26259.04 & $5 \mathrm{p}$ & $35 \mathrm{r}, \mathrm{II}$ & $a^{1} \overline{\mathrm{D}}_{2}-a^{3} \mathrm{D}_{2}$ & \\
\hline 67.505 & 830.95 & 5 & $12, \mathrm{II}$ & $a^{3} \bar{F}_{3}-a^{3} \bar{F}_{2}$ & & $3858.284 \mid 2$ & 25910.94 & $10 \mathrm{p}$ & $40 \mathrm{r}, \mathrm{II}$ & $a^{1} \overline{\mathrm{D}_{2}}-1 a^{3} \overline{\mathrm{F}}_{3}$ & \\
\hline 69.484 & 814.51 & 5 & $15, \mathrm{II}$ & $a^{3} \mathrm{~F}_{2}-a^{1} \overline{\mathrm{F}}_{3}$ & & & & & & & \\
\hline 72.545 & 789.11 & 50 & $70 \mathrm{R}, \mathrm{II}$ & $a^{3} \overline{\mathrm{D}}_{2}-a_{3} \mathrm{D}_{3}$ & & & & & & & \\
\hline 83. 776 & 696.30 & 20 & $25 \mathrm{R}, \mathrm{II}$ & $a^{3} \mathrm{~F}_{2}-a^{3} \mathrm{D}_{1}$ & & & & & & & \\
\hline 85.892 & 678.88 & 1 & $10, \mathrm{II}$ & & & & & & & & \\
\hline 3492.965 & 28620.81 & 150 & $150 \mathrm{R}, \mathrm{II}$ & $a^{3} \overline{\mathrm{D}}_{2}-a^{3} \overline{\mathrm{P}}_{1}$ & A & & & & & & \\
\hline
\end{tabular}

TABLE 6.-Low levels in the spectra of nickel

\begin{tabular}{|c|c|c|c|c|c|c|c|c|c|}
\hline $\begin{array}{l}\text { Spec- } \\
\text { trum }\end{array}$ & Value & $\begin{array}{c}\text { Separa- } \\
\text { tion }\end{array}$ & $\begin{array}{c}\text { Sym- } \\
\text { bol }\end{array}$ & Electrons & $\begin{array}{l}\text { Spec- } \\
\text { trum }\end{array}$ & Value & $\begin{array}{c}\text { Separa- } \\
\text { tion }\end{array}$ & $\begin{array}{c}\text { Sym- } \\
\text { bol }\end{array}$ & Electrons \\
\hline $\mathrm{Ni} \mathrm{I}$ & $\begin{array}{r}0.00 \\
204.82 \\
\\
879.82 \\
1332.15 \\
\\
1713.11 \\
\\
2216.55 \\
3409.95 \\
13521.29 \\
14728.92 \\
15609.81\end{array}$ & $\begin{array}{r}1332.15 \\
675.00 \\
884.40 \\
833.29 \\
\\
124.22\end{array}$ & $\begin{array}{l}a^{3} \mathrm{~F}_{4} \\
a^{3} \overline{\mathrm{D}}_{3} \\
a^{3} \overline{\mathrm{D}}_{2} \\
a^{3} \mathrm{~F}_{3} \\
a^{3} \overline{\mathrm{D}}_{1} \\
a^{3} \mathrm{~F}_{2} \\
a^{1} \overline{\mathrm{D}}_{2} \\
b^{1} \overline{\mathrm{D}}_{2} \\
a^{1} \mathrm{~S}_{0} \\
a^{3} \mathrm{P}_{2}\end{array}$ & $\begin{array}{l}4 s^{2}, 3 d^{3} \\
4 s, 3 d^{0}\end{array}$ & $\begin{array}{l}\text { Ni I } \\
\text { Ni II }\end{array}$ & $\begin{array}{r}15734.03 \\
16017.30 \\
0.0 \\
936.2 \\
1721.4 \\
\\
2269.7 \\
5156.3 \\
6601.2\end{array}$ & $\begin{array}{r}283.27 \\
\\
936.2 \\
785.2 \\
548.3 \\
\end{array}$ & $\begin{array}{l}a^{3} \mathrm{P}_{1} \\
a^{3} \mathrm{P}_{0} \\
a^{4} \mathrm{~F}_{5} \\
a^{4} \mathrm{~F}_{4} \\
a^{4} \mathrm{~F}_{3} \\
a^{4} \mathrm{~F}_{2} \\
a^{2} \mathrm{~F}_{4} \\
a^{2} \mathrm{~F}_{3}\end{array}$ & $4 s, 3 d^{8}$ \\
\hline
\end{tabular}




\section{DISCUSSION}

The results presented above confirm the conclusion already arrived at in other ways-that the normal state of the iron atom is represented by a quintet $\mathrm{D}$ spectroscopic term, that of cobalt by a quartet $\mathrm{F}$ term, and that of nickel by a triplet $\mathrm{F}$ term. Practically all of the absorbed lines involve either the normal state or some adjacent metastable state, and the fact that no prominent lines or groups of lines are unaccounted for indicates that the existence of other terms of lower energy is not probable. This indication is supported by the furnace absorption and by the astrophysical behavior of these elements, and it is, furthermore, in perfect accord with quantum-theoretical predictions. The latter are derived from a correlation of spectral terms with the electron configurations which produce them. Thus, for atoms with $z$ valence electrons in incomplete shells $(z=8,9,10$ for $\mathrm{Fe}, \mathrm{Co}, \mathrm{Ni}$, respectively) it has been shown that the following low levels are possible:

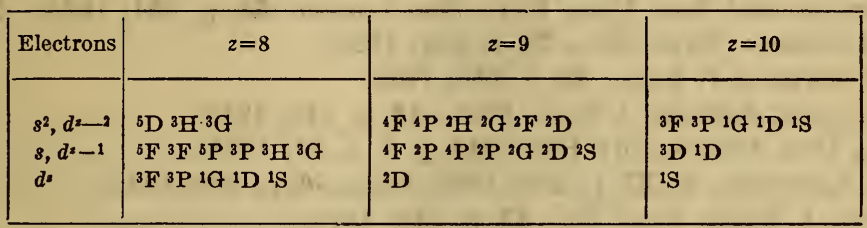

Each arrangement of electrons is responsible for a set of terms, the most prominent ones of which are given above, starting at the left with the term which presumably has the least energy. In each spectrum terms from all three configurations of electrons will appear, but at present the one which will give the normal state can only be determined empirically.

Comparing the absorption data for the three spectra-iron, cobalt, and nickel-we see that the lowest energy term arises in each case from the electron configuration of two $4 s$ electrons and $z-2$ electrons of $3 d$ type, but with increasing $z$ there is a progressive approach of the low metastable states to the normal one, and the lowest-energy term from the arrangement $4 s, 3 d^{z-1}$ acquires more and more prominence in absorption phenomena until in $\mathrm{Ni}$ it supersedes the normal state in this respect. Thus, the tendency for group configurations with a lesser number of $4 s$ electrons to be preferred, which was noticed in describing the normal states of the Ru-Rh-Pd and Os-Ir-Pt triads of metals (36), is apparently already in operation in the Fe-Co-Ni triad. 


\section{BIBLIOGRAPHY}

1. Wood, Astrophys. J., 29, p. 97; 1909. Bevan, Proc. Roy. Soc. London, 83, p. $421 ; 1910 ; 85$, p. 54 ; 1911 . Datta, Proc. Roy. Soc. London, 101, p. 539; 1922.

2. Grotrian, Zeitschr. f. Phys., 18, p. 169; 1923. (Pb.) Gieseler and Grotrian, Zeitschr. f. Phys. 25, p. 165; p. 342; 1924. (Fe, Mn, Sc, Ti, V.) King, Astrophys. J., 60, p. 282; 1924: (Cr.) Zumstein, Phys. Rev. 26, p. 765; 1925, 27, p. 106, p. 150; 1926. (Mn, Sn.) Sur and Majumdar, Phil. Mag. 1, p. $451 ; 1926$. (Al, Co.)

3. Angerer and Joos, Ann. der Phys., 74, p. 743; 1924.

4. Wilsing, Sitz. d. Akad. Wiss., Berlin, pp. 426-436; 1899.

5. Lockyer, Astrophys. J., 15, p. 190; 1902.

6. Hale, Astrophys. J., 15, p. 132; 1902.

7. Konen, Ann. der. Phys., 9, p. 742; 1902.

8. Finger, Zeitschr. f. Wiss. Phot., y, p. 329, p. 369; 1909.

9. E. and L. Bloch, J. de Phys. et le Rad., 3, p. 309; 1922.

10. Hulburt, Phys. Rev., 24, p. 129; 1924.

11. Stücklen, Zeitschr. f. Phys., 30, p. 24; 1924.

12. Sur, Phil. Mag., 1, p. 433; 1926. Kichlu, Zeitschr. f. Phys., 39, p. 572; 1926. Narayan and Rao, Proc. Phys. Soc. London, 38, p. 354; 1926. Meggers and Laporte, Phys. Rev., 28, p. 642; 1926.

13. Hund, Zeitschr. f. Phys., 33, p. 345; 1925.

14. Schumacher, Zeitschr. f. Wiss. Phot., 19, p. 149; 1919.

15. Burns, Lick. Observatory Bull., 24\%, p. 8, p. 27; 1913.

16. King, Astrophys. J., 37, p. 239; 1913; Ibid., 56, p. 318; 1922.

17. Walters, J. Wash. Acad. Sci., 13, p. 243; 1923.

18. Walters, J. O. S. A. and R. S. I., 8, p. 245; 1924 . Laporte, Zeitschr. f. Phys., 23, p. 135; 1924; Ibid., 26, p. 1; 1924. Meggers, Astrophys. J., 60, p. $60 ; 1924$.

19. Gieseler and Grotrian, Zeitschr. f. Phys., 22, p. 245; 1924.

20. Laporte, Nature, 11\%, p. 721; 1926.

21. Russell, Astrophys. J., 64, p. 194; 1926.

22. Piña, Anal. Soc. Esp. Fis. y. Quim., 16, p. 338; 1918.

23. Exner and Haschek, Spektren der Elemente, 2; 1911.

24. Dhein, Zeitschr. f. Wiss. Phot., 19, p. 289; 1920.

25. King, Astrophys. J., 42, p. 45; 1915.

26. Sur and Majumdar, Phil. Mag., 1, p. 451; 1926.

27. Walters, J. Wash. Acad. Sci., 14, p. 407; 1924.

28. Catalán and Bechert, Zeitschr. f. Phys., 32, p. 336, 1925.

29. Piña, Anal. Soc. Esp. Fis. y. Quim., 16, p. 338; 1918.

30. Hamm, Zeitschr. f. Wiss. Phot., 13, p. 105; 1913.

31. King, Astrophys. J., 42, p. 45; 1915.

32. Majumdar, Zeitschr. f. Phys., 39, p. 562; 1926.

33. Narayan and Rao, Proc. Phys. Soc. London, 38, p. 354; 1926.

34. Walters, J. Wash. Acad. Sci., 15, p. 88; 1925.

35. Bechert and Sommer, Ann. der Phys., 7\%, p. 351; 1925 . Bechert, Ann. der Phys., 7 \%, p. 537; 1925.

36. Meggers and Laporte, Phys. Rev., 28, p. 642; 1926.

Washington, February 1, 1927. 